\title{
Algorithms for bispectra: forecasting, optimal analysis, and simulation
}

\author{
Kendrick M. Smith \\ Kavli Institute for Cosmological Physics, University of Chicago, 60637 and \\ Department of Physics, University of Chicago, 60637 \\ Matias Zaldarriaga \\ Institute for Theory and Computation, Harvard-Smithsonian Center for Astrophysics, \\ MS-51, 60 Garden Street, Cambridge, MA 02138, USA and \\ Jefferson Laboratory of Physics, Harvard University, Cambridge, Massachusetts 02138, USA
}

\begin{abstract}
We propose a factorizability ansatz for angular bispectra which permits fast algorithms for forecasting, analysis, and simulation, yet is general enough to encompass many interesting CMB bispectra. We describe a suite of general algorithms which apply to any bispectrum which can be represented in factorizable form. First, we present algorithms for Fisher matrix forecasts and the related problem of optimizing the factorizable representation, giving a Fisher forecast for Planck as an example. We show that the CMB can give independent constraints on the amplitude of primordial bispectra of both local and equilateral shape as well as those created by secondary anisotropies. We also show that the ISW-lensing bispectrum should be detected by Planck and could bias estimates of the local type of non-Gaussianity if not properly accounted for. Second, we implement a bispectrum estimator which is fully optimal in the presence of sky cuts and inhomogeneous noise, extends the generality of fast estimators which have been limited to a few specific forms of the bispectrum, and improves the running time of existing implementations by several orders of magnitude. Third, we give an algorithm for simulating random, weakly non-Gaussian maps with prescribed power spectrum and factorizable bispectrum.
\end{abstract}

\section{INTRODUCTION}

Despite the rapid progress in observational cosmology in the last decade, there are very few observational probes that are able to constrain the first instants in the evolution of the Universe, when density perturbations were created. In addition to the shape of the primordial spectrum of perturbations and the amplitude of the gravitational wave background, any measurable departure from pure Gaussianity in the statistics of the primordial seeds would severely constrain the inflationary dynamics. The level of non-Gaussianity in the simplest, single field, inflationary models has now been robustly calculated and shown to be too small to be measured by upcoming Cosmic Microwave Background (CMB) experiments [1, 2]. Various departures from the simplest scenario however are thought to produce observable signals $[3-10]$.

In fact it has become clear that the three point function of the primordial fluctuations, the bispectrum, is the most promising statistic to probe the small departures from Gaussianity that could originate during inflation 11]. Moreover the structure of the three point function contains precious information about the inflationary dynamics [12]. For example as a result of causality, the three point function in models where only one field is effectively responsible for inflation have to satisfy strict consistency relations that constrain the configurational dependance of the bispectrum 1, 13]. Furthermore we have learned that the shape of the bispectrum of any primordial non-Gaussianity arising in inflationary models neatly falls in two separate classes. The momentum space three point function in single field models is largest when the three momenta are of similar magnitude, while for multi-field models where curvature perturbations are created by a field different from the one that drives inflation, the bispectrum is largest in the squeezed limit, when one of the momenta is much smaller than the other two.

If we are to probe the primordial non-Gaussianities using the CMB we also need to consider the departures from Gaussianity produced by secondary anisotropies as well as residual foreground contamination (eg. [14 17]). In general the non-linear dynamics of Gravity and of any probe we wish to use will lead to some departures from Gaussianity which are not of primordial origin. These additional sources on non-Gaussianity produce bispectra with specific configurational dependance.

It is clear then that if we want to hunt for possible departures from Gaussainity as well as constrain the evolution of perturbations after decoupling through secondary anisotropies, we need to develop data analysis tools that will allow us to measure the bispectrum efficiently and can distinguish between different shapes of the momentum space bispectrum. The efficiency of the tools will be crucial as the expected level of non-Gaussianity is rather small so it will only be detectable in large surveys, with a large number of pixels. Developing these tools is the object of this paper. The effort is timely as many of the predicted signals are expected to be detectable by the upcoming Planck satellite.

In spite of the promise offered by the three-point function, and the variety of forms that have been calculated, there is currently a lack of general methods for connecting a predicted three-point function with data. The basic problem is that the most general three-point function al- 
lowed by rotational invariance is described by its angular bispectrum $B_{\ell_{1} \ell_{2} \ell_{3}}$, an object with $\mathcal{O}\left(\ell_{\max }^{3}\right)$ degrees of freedom. In this generality, algorithms tend to be prohibitively expensive; for example, evaluating an optimal bispectrum estimator has cost $\mathcal{O}\left(\ell_{\max }^{5}\right)$. Computationally feasible algorithms are only known for a few special forms of the bispectrum [18, 19]. One might make an analogy with power spectrum estimation; many solutions have been proposed to the problem of optimally estimating power spectra from data 20 28], but given a prediction for the shape of the bispectrum which is to be estimated from data, relatively little is known.

The purpose of this paper is to address this problem by proposing a "toolkit" of algorithms for forecasting, optimal estimation, and non-Gaussian simulation. These fast algorithms will apply to any bispectrum which can be represented in a factorizable form which will be defined below (Eq. (3)). We will show (\$III) that a wide range of previously calculated CMB bispectra can be represented in this form, thus giving wide scope to our methods. Our methods do not apply to a completely arbitrary bispectrum $B_{\ell_{1} \ell_{2} \ell_{3}}$, but we believe this to be a necessary feature of any treatment which leads to fast algorithms. The factorizability criterion is a compromise in generality which is specific enough to enable practical computation, yet general enough to encompass a large number of interesting cases.

Our first algorithm ( $₫ \sqrt{\mathrm{IV}}$ ) attempts to "optimize" a bispectrum by reducing the size of its factorizable representation. We will see that this is closely related to computing a Fisher matrix forecast under the assumption of homogeneous noise. The optimization algorithm can be used as a preliminary step to speed up the other algorithms; we will also see examples where a bispectrum with an intractably large factorizable representation is optimized down to manageable size, thus giving additional scope to our methods. As an application, we will present ( $(\overline{\mathrm{VI}})$ a multi-bispectrum Fisher matrix forecast for Planck.

Second, we give a general Monte Carlo framework for estimating bispectra from noisy data, in the presence of sky cuts and inhomogeneous noise (VII-\$VIII). This generalizes the estimator proposed by Komatsu, Spergel, and Wandelt [18] to arbitrary factorizable bispectra, and also improves it in the case of inhomogenous noise, by including the linear term in the estimator proposed in [19]. We also present some code optimizations which dramatically improve existing implementations and make Monte Carlo estimation practical for Planck.

Third, we give a simulation algorithm ( $₫$ IX) which outputs random non-Gaussian maps with arbitrary power spectrum and factorizable bispectrum. This greatly extends the generality of existing methods for simulating non-Gaussian fields 29 32].

Throughout this paper, we make the assumption of weak non-Gaussianity. On a technical level, this means that the covariance of the three-point function is well approximated by its Gaussian contribution. If this ap- proximation breaks down, then both the choice of optimal estimator and the estimator variance can be affected. It might seem that the CMB is so close to Gaussian that this is never an issue; however, it has recently been shown [11] that it can be important for bispectra of "squeezed" shape, if a several-sigma detection can be made, even though the field is still very close to Gaussian. A second reason that the assumption of weak nonGaussianity might break down is that the lensed CMB is non-Gaussian; e.g. in [33], it is argued that this may degrade constraints on $f_{N L}$ by $\sim 25 \%$ at Planck sensitivity. Both of these effects are outside the scope of this paper.

\section{NOTATION AND CONVENTIONS}

The angular CMB bispectrum $B_{\ell_{1} \ell_{2} \ell_{3}}$ is defined by

$$
\left\langle a_{\ell_{1} m_{1}} a_{\ell_{2} m_{2}} a_{\ell_{3} m_{3}}\right\rangle=B_{\ell_{1} \ell_{2} \ell_{3}}\left(\begin{array}{ccc}
\ell_{1} & \ell_{2} & \ell_{3} \\
m_{1} & m_{2} & m_{3}
\end{array}\right) .
$$

where the quantity in parentheses is the Wigner $3 \mathrm{j}$ symbol. This is the most general three-way expectation value which is invariant under rotations. Following Komatsu and Spergel [34], we define the reduced bispectrum $b_{\ell_{1} \ell_{2} \ell_{3}}$ by

$$
\begin{array}{r}
B_{\ell_{1} \ell_{2} \ell_{3}}=\left[\frac{\left(2 \ell_{1}+1\right)\left(2 \ell_{2}+1\right)\left(2 \ell_{3}+1\right)}{4 \pi}\right]^{1 / 2} \\
\times\left(\begin{array}{ccc}
\ell_{1} & \ell_{2} & \ell_{3} \\
0 & 0 & 0
\end{array}\right) b_{\ell_{1} \ell_{2} \ell_{3} .} .
\end{array}
$$

Because the $3 \mathrm{j}$-symbol on the right-hand side vanishes for triples $\left(\ell_{1}, \ell_{2}, \ell_{3}\right)$ such that $\left(\ell_{1}+\ell_{2}+\ell_{3}\right)$ is odd, Eq. (2) only makes sense if $B_{\ell_{1} \ell_{2} \ell_{3}}$ also vanishes for such triples. This condition is equivalent to parity invariance of the bispectrum, and will be satisfied for all bispectra considered in this paper.

\section{FACTORIZABILITY}

A basic problem in the theory of bispectrum estimation is that there are so many degrees of freedom in the bispectrum $B_{\ell_{1} \ell_{2} \ell_{3}}$ that completely general methods, which make no assumption on the form of $B_{\ell_{1} \ell_{2} \ell_{3}}$, are computationally prohibitive. For example, even with the unrealistic assumption of homogeneous instrumental noise, the cost of evaluating an optimal estimator is $\mathcal{O}\left(\ell_{\max }^{5}\right)$ [35].

In this section, we propose a factorizability ansatz for the form of the bispectrum, and show that many CMB bispectra of theoretical interest can be written in factorizable form. Our approach is empirical; we simply collect and analyze interesting bispectra from the literature. In subsequent sections, we will present fast algorithms which can be applied to factorizable bipsectra, 
which will improve the $\mathcal{O}\left(\ell_{\max }^{5}\right)$ cost and make calculations tractable.

Our ansatz is that the reduced bispectrum defined in Eq. (2) is a sum of terms factorizable in $\ell_{1}, \ell_{2}, \ell_{3}$ :

$$
b_{\ell_{1} \ell_{2} \ell_{3}}=\frac{1}{6} \sum_{i=1}^{N_{\text {fact }}} X_{\ell_{1}}^{(i)} Y_{\ell_{2}}^{(i)} Z_{\ell_{3}}^{(i)}+\text { (symm.) }
$$

where $N_{\text {fact }}$ is not too large. In Eq. (3) and throughout the paper, + (symm.) stands for the sum of five additional terms obtained by permuting $\ell_{1}, \ell_{2}, \ell_{3}$.

\section{A. CMB secondaries}

The first general class of bispectra which satisfy the factorizability condition (Eq. (3)) are those which arise from three-way correlations between the primary CMB, lensed CMB, and secondary anisotropies [14, 15]. These are of a manifestly factorizable (with $N_{\text {fact }}=3$ ) form,

$$
b_{\ell_{1} \ell_{2} \ell_{3}}=\frac{\ell_{1}\left(\ell_{1}+1\right)-\ell_{2}\left(\ell_{2}+1\right)+\ell_{3}\left(\ell_{3}+1\right)}{2} C_{\ell_{1}} \beta_{\ell_{3}}
$$

where $\beta_{\ell}$ depends on the secondary anisotropy which is considered.

For example, with secondary anisotropy given by the integrated Sachs-Wolfe (ISW) effect, $\beta_{\ell}$ is equal to $C_{\ell}^{T \phi}$, the cross power spectrum between the unlensed CMB temperature and lensing potential. In this case, the bispectrum should be detectable by Planck [15, 36], and would provide a direct signature, internal to the CMB, of an evolving gravitational potential [37]. We will refer to this as the ISW-lensing bispectrum and use it as a running example throughout the paper. Other examples of the general form in Eq. (4) have also been studied; e.g. the Rees-Sciama-lensing bispectrum [16], and the SZ-lensing bispectrum [15, 17].

Another general class of $\mathrm{CMB}$ bispectra is given by three-way correlations between Ostriker-Vishniac anisotropies and secondary anisotropies [17, 38]. These bispectra are of the form:

$$
b_{\ell_{1} \ell_{2} \ell_{3}}=\int d r f_{\ell_{1}}(r) g_{\ell_{2}}(r)+(\text { symm. })
$$

To make this factorizable, we replace the $r$ integral by a finite quadrature:

$$
b_{\ell_{1} \ell_{2} \ell_{3}} \rightarrow \sum_{i=1}^{N_{\text {fact }}}\left(\Delta r_{i}\right) f_{\ell_{1}}\left(r_{i}\right) g_{\ell_{2}}\left(r_{i}\right)+\text { (symm.) }
$$

The bispectrum is then of the factorizable form (Eq. (3)), where $N_{\text {fact }}$ is the number of quadrature points needed to approximate the integral. This device, replacing an integral by a finite sum in order to satisfy the factorizability condition, will be used frequently throughout the paper.
Finally, we mention a mathematically trivial but practically important example: the bispectrum from residual point sources (assumed Poisson distributed) is given by

$$
b_{\ell_{1} \ell_{2} \ell_{3}}^{\mathrm{ps}}=\text { constant }
$$

The value of $b^{\mathrm{ps}}$ will depend on the flux limit at which point sources can be detected and masked in the survey considered, and on the assumed flux and frequency distribution of the sources. As a rough baseline, motivated by [35, 39], we will take $b_{\ell_{1} \ell_{2} \ell_{3}}^{\mathrm{ps}}=10^{-8} \mu \mathrm{K}^{3}$ at Planck sensitivity, corresponding to a flux limit $\sim 0.2$ Jy at 217 $\mathrm{GHz}$.

\section{B. Primordial non-Gaussianity}

Moving on, we next consider CMB bispectra which arise from primoridal non-Gaussianity, rather than secondary anisotropies. The general relation between the angular CMB bispectrum and primordial three-point function can be described as follows. We assume that the primordial three-point function is invariant under translations and rotations, so that three-way correlations in the Newtonian potential $\Phi$ are given by

$\left\langle\Phi\left(\mathbf{k}_{1}\right) \Phi\left(\mathbf{k}_{2}\right) \Phi\left(\mathbf{k}_{3}\right)\right\rangle=(2 \pi)^{3} \delta^{3}\left(\mathbf{k}_{1}+\mathbf{k}_{2}+\mathbf{k}_{3}\right) F\left(k_{1}, k_{2}, k_{3}\right)$

where $F$ depends only on the lengths $k_{i}=\left|\mathbf{k}_{i}\right|$, as implied by the notation. In [40], it is shown that the reduced angular bispectrum is given by

$$
\begin{gathered}
b_{\ell_{1} \ell_{2} \ell_{3}}=\int d r r^{2}\left(\prod_{i=1}^{3} \frac{2 k_{i}^{2} d k_{i}}{\pi} j_{\ell_{i}}\left(k_{i} r\right) \Delta_{\ell_{i}}^{T}\left(k_{i}\right)\right) \\
\times F\left(k_{1}, k_{2}, k_{3}\right)
\end{gathered}
$$

where $\Delta_{\ell_{i}}^{T}(k)$ denotes the transfer function between the CMB multipoles and the Newtonian potential:

$$
a_{\ell m}=4 \pi(i)^{\ell} \int \frac{d^{3} \mathbf{k}}{(2 \pi)^{3}} \Delta_{\ell}^{T}(k) \Phi(\mathbf{k}) Y_{\ell m}^{*}(\hat{\mathbf{k}})
$$

Now let us consider some specific examples of primordial non-Gaussianity. The simplest is the "local model", in which the primordial potential satisfies:

$$
\Phi(\mathbf{x})=\Phi_{G}(\mathbf{x})-f_{N L}^{\text {loc }}\left(\Phi_{G}(\mathbf{x})^{2}-\left\langle\Phi_{G}(\mathbf{x})^{2}\right\rangle\right)
$$

where $f_{N L}^{\text {loc }}$ is a constant and $\Phi_{G}$ is Gaussian. In this model, the primordial bispectrum is

$$
F^{\mathrm{loc}}\left(k_{1}, k_{2}, k_{3}\right)=f_{N L}^{\mathrm{loc}}\left(\frac{\Delta_{\Phi}^{2}}{k_{1}^{4-n_{s}} k_{2}^{4-n_{s}}}\right)+(\text { symm. })
$$

where $f_{N L}^{\text {loc }}$ is a constant and $P^{\Phi}(k)=\Delta_{\Phi} / k^{4-n_{s}}$ is the primordial power spectrum. Substituting Eq. (12) into Eq. (9), the angular CMB bispectrum is

$$
b_{\ell_{1} \ell_{2} \ell_{3}}^{\text {loc }}=f_{N L}^{\text {loc }} \int d r r^{2} \beta_{\ell_{1}}(r) \beta_{\ell_{2}}(r) \alpha_{\ell_{3}}(r)+\text { (symm.) }
$$


where, following [34], we have introduced the functions

$$
\begin{aligned}
& \alpha_{\ell}(r)=\frac{2}{\pi} \int_{0}^{\infty} d k k^{2} j_{\ell}(k r) \Delta_{\ell}^{T}(k) \\
& \beta_{\ell}(r)=\frac{2}{\pi} \int_{0}^{\infty} d k k^{2} j_{\ell}(k r) \Delta_{\ell}^{T}(k)\left(\frac{\Delta_{\Phi}}{k^{4-n_{s}}}\right)
\end{aligned}
$$

After replacing the $r$ integral in Eq. (13) by a finite quadrature, the local bispectrum is of factorizable form (3), with $N_{\text {fact }}$ equal to the number of quadrature points.

This illustrates a general point: suppose that the primordial bispectrum $F\left(k_{1}, k_{2}, k_{3}\right)$ is a sum of $M$ terms each factorizable in $k_{1}, k_{2}, k_{3}$. Then the resulting CMB bispectrum $b_{\ell_{1} \ell_{2} \ell_{3}}$ will be a sum of $N_{\text {fact }}=M N_{\text {quad }}$ terms each factorizable in $\ell_{1}, \ell_{2}, \ell_{3}$, where $N_{\text {quad }}$ is the number of quadrature points need to do the $r$ integral in Eq. (9).

Our next example is taken from [41], in which a primordial bispectrum of the form

$$
\begin{aligned}
F^{\text {grav }}\left(\mathbf{k}_{1}, \mathbf{k}_{2}, \mathbf{k}_{3}\right) & =\frac{\Delta_{\Phi}^{2}}{k_{1}^{4-n_{s}} k_{2}^{4-n_{s}}} f_{N L}^{\text {grav }}\left(\mathbf{k}_{1}, \mathbf{k}_{2}, \mathbf{k}_{3}\right) \\
f_{N L}^{\text {grav }}\left(\mathbf{k}_{1}, \mathbf{k}_{2}, \mathbf{k}_{3}\right) & =-\frac{1}{6}-\frac{\mathbf{k}_{1} \cdot \mathbf{k}_{2}}{k_{3}^{2}}+\frac{3\left(\mathbf{k}_{1} \cdot \mathbf{k}_{3}\right)\left(\mathbf{k}_{2} \cdot \mathbf{k}_{3}\right)}{k_{3}^{4}}
\end{aligned}
$$

is studied, arising from second-order gravitational evolution after inflation [51]. Using the constraint $\left(\mathbf{k}_{1}+\mathbf{k}_{2}+\right.$ $\left.\mathbf{k}_{3}\right)=0$ from Eq. (8), we rewrite $f_{N L}^{\text {grav }}$ in factorizable form

$$
f_{N L}^{\text {grav }}\left(k_{1}, k_{2}, k_{3}\right)=\frac{1}{12}+\frac{k_{1}^{2}}{k_{3}^{2}}-\frac{3 k_{1}^{4}}{2 k_{3}^{4}}+\frac{3 k_{1}^{2} k_{2}^{2}}{2 k_{3}^{4}} .
$$

The resulting CMB bispectrum $b_{\ell_{1} \ell_{2} \ell_{3}}^{\text {grav }}$ will then be of factorizable form (Eq. (3)), with $N_{\text {fact }}=4 N_{\text {quad }}$, where $N_{\text {quad }}$ is the number of quadrature points needed to do the $r$ integral in Eq. (9).

This example illustrates the ubiquity and power of the factorizability ansatz. In [41], finding a computationally feasible method for computing Fisher matrix uncertainties for the gravitational bispectrum at Planck noise levels was left as an unsolved problem. After representing the bispectrum in factorizable form, we will find, using the algorithms to be presented in the rest of the paper, that in addition to computing the Fisher matrix rapidly, we can compute an optimal estimator and construct nonGaussian simulated maps for this bispectrum.

Next, we consider the "higher derivative model" from [12], which arises from adding the higher derivative operator $(\nabla \phi)^{4} /\left(8 \Lambda^{4}\right)$ to the inflationary Lagrangian [4, [] ]

$$
\begin{aligned}
F^{\mathrm{hd}}\left(k_{1}, k_{2}, k_{3}\right) & =\frac{9}{7} f_{N L}^{\mathrm{hd}} \Delta_{\Phi}^{2}\left[-k_{1}^{-3} k_{2}^{-3}\right. \\
& \left.+8 \frac{k_{1}^{-3} k_{2}^{-1}+k_{1}^{-2} k_{2}^{-1} k_{3}^{-1}}{\left(k_{1}+k_{2}+k_{3}\right)^{2}}\right]+(\text { symm. })
\end{aligned}
$$

This expression assumes scale invariance. In the general case, the amplitude of the three point function depends on the dynamics of the field and the expansion of the
Universe at the time the triangle of interest crosses the horizon during inflation. For equilateral configurations all three modes in the triangle cross the horizon at approximately the same time.

Following a standard convention, we have introduced a parameter $f_{N L}^{\text {hd }}$ for the amplitude of the bispectrum, normalized so that with all three momenta equal, $F(k, k, k)=6 f_{N L}^{\text {hd }}\left(\Delta_{\Phi} / k^{3}\right)^{2}$. The value of $f_{N L}^{\text {hd }}$ is given by $f_{N L}^{\text {hd }}=(35 / 432) \dot{\phi}^{2} / \Lambda^{4}$, where $\dot{\phi}$ is the velocity of the inflaton. (The same bispectrum also arises, typically with a larger value of $f_{N L}^{\text {hd }}$, for DBI inflation [6].)

The factor $1 /\left(k_{1}+k_{2}+k_{3}\right)^{2}$ appears to ruin factorizability; however, this disease can be cured by introducing a Schwinger parameter $t$ and writing

$$
\frac{1}{\left(k_{1}+k_{2}+k_{3}\right)^{2}}=\int_{0}^{\infty} d t t e^{-t\left(k_{1}+k_{2}+k_{3}\right)}
$$

Using Eqs. (19), (9), one arrives at a CMB bispectrum of the form:

$$
\begin{array}{r}
b_{\ell_{1} \ell_{2} \ell_{3}}^{\mathrm{hd}}=\frac{9}{7} f_{N L}^{\mathrm{hd}}\left[-\int_{0}^{\infty} d r r^{2} \beta_{\ell_{1}}(r) \beta_{\ell_{2}}(r) \alpha_{\ell_{3}}(r)\right. \\
+8 \Delta_{\Phi}^{2 / 3} \int_{0}^{\infty} d t t \int_{0}^{\infty} d r r^{2}\left(\beta_{\ell_{1}}(r, t) \gamma_{\ell_{2}}(r, t) \alpha_{\ell_{3}}(r, t)\right. \\
\left.\left.+\delta_{\ell_{1}}(r, t) \gamma_{\ell_{2}}(r, t) \gamma_{\ell_{3}}(r, t)\right)\right]+(\text { symm. })
\end{array}
$$

where we have defined:

$$
\begin{aligned}
& \alpha_{\ell}(r, t)=\frac{2}{\pi} \int_{0}^{\infty} d k k^{2} j_{\ell}(k r) \Delta_{\ell}^{T}(k) e^{-t k} \\
& \beta_{\ell}(r, t)=\frac{2}{\pi} \int_{0}^{\infty} d k k^{2} j_{\ell}(k r) \Delta_{\ell}^{T}(k) e^{-t k}\left(\frac{\Delta_{\Phi}}{k^{3}}\right) \\
& \gamma_{\ell}(r, t)=\frac{2}{\pi} \int_{0}^{\infty} d k k^{2} j_{\ell}(k r) \Delta_{\ell}^{T}(k) e^{-t k}\left(\frac{\Delta_{\Phi}}{k^{3}}\right)^{1 / 3} \\
& \delta_{\ell}(r, t)=\frac{2}{\pi} \int_{0}^{\infty} d k k^{2} j_{\ell}(k r) \Delta_{\ell}^{T}(k) e^{-t k}\left(\frac{\Delta_{\Phi}}{k^{3}}\right)^{2 / 3}
\end{aligned}
$$

(This ordering was chosen so that, for $t=0$, these reduce to the functions $\alpha_{\ell}(r), \beta_{\ell}(r)$ defined in Eq. (14) and the functions $\gamma_{\ell}(r), \delta_{\ell}(r)$ defined in [19].) Note that we have assumed scale invariance throughout our treatment of the higher-derivative bispectrum. It is seen that the bispectrum is of factorizable form (Eq. (3)), with $N_{\text {fact }}=N_{1}+2 N_{2}$, where $N_{1}, N_{2}$ are the numbers of quadrature points needed to do the single and double integrals in Eq. (20).

Finally, following [19], we introduce the "equilateral" bispectrum

$$
\begin{gathered}
F^{\mathrm{eq}}\left(k_{1}, k_{2}, k_{3}\right)=f_{N L}^{\mathrm{eq}}\left[-3 \frac{\Delta_{\Phi}^{2}}{k_{1}^{4-n_{s}} k_{2}^{4-n_{s}}}\right. \\
-2 \frac{\Delta_{\Phi}^{2}}{k_{1}^{2\left(4-n_{s}\right) / 3} k_{2}^{2\left(4-n_{s}\right) / 3} k_{3}^{2\left(4-n_{s}\right) / 3}} \\
\left.+6 \frac{\Delta_{\Phi}^{2}}{k_{1}^{\left(4-n_{s}\right) / 3} k_{2}^{2\left(4-n_{s}\right) / 3} k_{3}^{4-n_{s}}}\right]+(\text { symm. })
\end{gathered}
$$


In contrast to the other bispectra discussed so far, this bispectrum does not arise from a model; rather it is designed to approximate the higher-derivative bispectrum (Eq. (19) ) using fewer factorizable terms. In [19], it was shown that the two bispectra are so highly correlated that it suffices to work with $F^{\text {eq }}$ for data analysis purposes. We will confirm this result in $\S \mathrm{VI}$. The equilateral bispectrum is manifestly of factorizable form, with $N_{\text {fact }}=3 N_{\text {quad }}$, where $N_{\text {quad }}$ is the number of quadrature points needed to do the $r$ integral (Eq. (9)).

As the name suggests, for the equilateral bispectrum (Eq. (22) ), most of the signal-to-noise is contributed by triples $\left(\ell_{1}, \ell_{2}, \ell_{3}\right)$ for which the $\ell$ 's are of comparable magnitude. In contrast, for the local bispectrum (Eq. (12)), the greatest contribution is from "squeezed" triangles in which $\ell_{1} \ll \ell_{2}, \ell_{3}$. This is reflected in the asymptotics of the $3 \mathrm{D}$ bispectra in the squeezed limit $k_{2}=k_{3}, k_{1} \rightarrow 0$. The leading behavior of the local bispectrum is $\mathcal{O}\left(k_{1}^{-3}\right)$, whereas the higher-derivative (Eq. (19)) and equilateral bispectra have leading behavior $\mathcal{O}\left(k_{1}^{-1}\right)$ arising from a cancellation between terms. The gravitational bispectrum (Eq. (16) ) has the same $\mathcal{O}\left(k_{1}^{-3}\right)$ behavior as the local bispectrum.

\section{FISHER MATRIX}

Before discussing inhomogeneous noise, we first consider the simplest possible noise model for a survey: homogeneous parameterized by noise power spectrum $N_{\ell}$. Such a noise model can be used as an approximation when forecasting sensitivity to different bispectra. In this noise model, the Fisher matrix of bispectra $B_{1}, B_{2}, \ldots$ is defined by

$$
F_{\alpha \beta} \stackrel{\text { def }}{=} \frac{1}{6} \sum_{\ell_{1} \ell_{2} \ell_{3}} \frac{\left(B_{\alpha}\right) \ell_{1} \ell_{2} \ell_{3}\left(B_{\beta}\right)_{\ell_{1} \ell_{2} \ell_{3}}}{C_{\ell_{1}} C_{\ell_{2}} C_{\ell_{3}}}
$$

where $C_{\ell}=C_{\ell}^{T T}+N_{\ell}$ is the total signal + noise power spectrum. (In Eq. (23), we have written the Fisher matrix for an all-sky survey; for partial sky coverage, one makes the approximation that the Fisher matrix scales as $\left.F_{\alpha \beta}\left(f_{\text {sky }}\right) \propto f_{\text {sky }} F_{\alpha \beta}(1).\right)$

The bispectrum covariance obtained from the survey is given by the inverse Fisher matrix:

$$
\operatorname{Cov}\left(B_{\alpha}, B_{\beta}\right)=\left(F^{-1}\right)_{\alpha \beta}
$$

(In particular, the marginalized $1 \sigma$ error on bispectrum $B_{\alpha}$ is given by $\sigma_{\operatorname{marg}}\left(B_{\alpha}\right)=\left(F^{-1}\right)_{\alpha \alpha}^{1 / 2}$ while the error with the other bispectra fixed is given by $\sigma_{\text {fixed }}\left(B_{\alpha}\right)=$ $\left(F_{\alpha \alpha}\right)^{-1 / 2}$.) Thus, the Fisher matrix gives a complete forecast for bispectrum sensitivity of a given survey, including cross-correlation information, under the simplifying assumption of homogeneous noise.

Let us consider the problem of efficient evaluation of the Fisher matrix in Eq. (23), assuming that the bispectra under consideration satisfy the factorizability condition from $₫$ III.
A "brute force" harmonic-space approach is to simply perform the sum over all triples $\left(\ell_{1}, \ell_{2}, \ell_{3}\right)$ given in Eq. (23), evaluating the bispectra $\left(B_{\alpha}\right) \ell_{1} \ell_{2} \ell_{3}$ by straightforward use of Eqs. (2), (3). The computational cost of this approach is $\mathcal{O}\left(N_{\text {fact }} \ell_{\max }^{3}\right)$. In many cases, we have found that the harmonic-space approach gives reasonable performance and allows the Fisher matrix to be computed straightforwardly.

A second approach is based on computing the Fisher matrix in position space rather than harmonic space. For notational simplicity we will present the method for the case of a single bispectrum (so that the Fisher matrix reduces to a number $F$ ) but our method extends straightforwardly to the multi-bispectrum case. Writing out the Fisher matrix,

$$
\begin{aligned}
F= & \frac{1}{6} \sum_{\ell_{1} \ell_{2} \ell_{3}} \frac{\left(B_{\ell_{1} \ell_{2} \ell_{3}}\right)^{2}}{C_{\ell_{1}} C_{\ell_{2}} C_{\ell_{3}}} \\
= & \sum_{\ell_{1} \ell_{2} \ell_{3} i j} \frac{\left(2 \ell_{1}+1\right)\left(2 \ell_{2}+1\right)\left(2 \ell_{3}+1\right)}{144 \pi}\left(\begin{array}{ccc}
\ell_{1} & \ell_{2} & \ell_{3} \\
0 & 0 & 0
\end{array}\right)^{2} \\
& \left.\times \frac{X_{\ell_{1}}^{(i)} Y_{\ell_{2}}^{(i)} Z_{\ell_{3}}^{(i)}}{C_{\ell_{1}} C_{\ell_{2}} C_{\ell_{3}}}\left[X_{\ell_{1}}^{(j)} Y_{\ell_{2}}^{(j)} Z_{\ell_{3}}^{(j)}+\text { (symm. }\right)\right]
\end{aligned}
$$

we use the identity

$$
\int_{-1}^{1} d z P_{\ell_{1}}(z) P_{\ell_{2}}(z) P_{\ell_{3}}(z)=2\left(\begin{array}{ccc}
\ell_{1} & \ell_{2} & \ell_{3} \\
0 & 0 & 0
\end{array}\right)^{2}
$$

to write $F$ in the form

$$
F=\sum_{i, j=1}^{N_{\text {fact }}} F_{i j}
$$

where we have defined

$$
F_{i j} \stackrel{\text { def }}{=} \frac{2 \pi^{2}}{9} \int_{-1}^{1}\left[\zeta_{X^{(i)} X^{(j)}} \zeta_{Y^{(i)} Y^{(j)}} \zeta_{Z^{(i)} Z^{(j)}}+\text { (perm.) }\right]
$$

where + (perm.) denotes the sum of five additional terms obtained by permuting $\left\{X^{(j)}, Y^{(j)}, Z^{(j)}\right\}$ and

$$
\zeta_{X Y}(z) \stackrel{\text { def }}{=} \sum_{\ell} \frac{(2 \ell+1)}{4 \pi} \frac{X_{\ell} Y_{\ell}}{C_{\ell}} P_{\ell}(z)
$$

where $P_{\ell}(z)$ denotes the Legendre polynomial.

To turn this into an algorithm for computing $F$, we note that the integral in Eq. (29) can be done exactly, using Gauss-Legendre integration [42] with $N_{\text {quad }}=$ $\left\lfloor 3 \ell_{\max } / 2\right\rfloor+1$ quadrature points, since the integrand is a polynomial in $z$ whose degree is $\leq 3 \ell_{\max }$. We loop over quadrature points $z$, computing the value of each function $\zeta_{X Y}(z)$ which appears, and accumulating the contribution to each $F_{i j}$ from point $z$, before moving on to the next quadrature point. This gives a position-space algorithm for Fisher matrix evaluation whose computational cost is $\mathcal{O}\left(N_{\text {fact }}^{2} \ell_{\text {max }}^{2}\right)$. As a rough rule of thumb, 
we have found that this position-space method is faster when $N_{\text {fact }} \lesssim 2 \ell_{\text {max }}$, and the $\mathcal{O}\left(N_{\text {fact }} \ell_{\max }^{3}\right)$ harmonicspace method is faster when $N_{\text {fact }} \gtrsim 2 \ell_{\max }$, but the constant which appears here will depend on the implementation.

We have introduced the matrix $F_{i j}$ as a device for computing the 1-by-1 "matrix" $F$, by summing the entries as in Eq. (28), but we note that $F_{i j}$ has a direct interpretation as the $N_{\text {fact }}$-by- $N_{\text {fact }}$ Fisher matrix of the individual terms in the factorizable bispectrum (Eq. (3)). This observation will be important for the optimization algorithm which we now present.

\section{OPTIMIZING $N_{\text {fact }}$}

From the preceding discussion, it may seem that our position-space method for Fisher matrix evaluation is of limited usefulness, providing significant speedup over the harmonic-space method only in the regime $N_{\text {fact }} \ll \ell_{\text {max }}$. However, as we will see in this section, the position-space method also leads to a means of "optimizing" a bispectrum written as a sum of many factorizable terms:

$$
b_{\ell_{1} \ell_{2} \ell_{3}}=\frac{1}{6} \sum_{i=1}^{N_{\text {fact }}} X_{\ell_{1}}^{(i)} Y_{\ell_{2}}^{(i)} Z_{\ell_{3}}^{(i)}+(\text { symm. })
$$

by approximating $b_{\ell_{1} \ell_{2} \ell_{3}}$ by a factorizable bispectrum with fewer terms. We present an algorithm which retains a subset (of size $N_{\text {opt }}$ ) of the original terms and chooses weights $w_{1}, \ldots, w_{N_{\mathrm{opt}}}$ such that the bispectrum

$$
b_{\ell_{1} \ell_{2} \ell_{3}}^{\prime}=\frac{1}{6} \sum_{i=1}^{N_{\mathrm{opt}}} w_{i} X_{\ell_{1}}^{(i)} Y_{\ell_{2}}^{(i)} Z_{\ell_{3}}^{(i)}+\text { (symm.) }
$$

is a good approximation to $b$. (In Eq. (32) and throughout this section, we have assumed for notational simplicity that the terms in the original bispectrum (Eq. (31)) have been reordered so that the terms to be retained are in positions $1, \ldots, N_{\text {opt }}$.)

Generally speaking, it is only possible to optimize a bispectrum by exploiting redundancy in the factorizable representation, such as consecutive terms which are nearly equal, or terms which are small enough to be negligible. For example, a randomly generated factorizable bispectrum cannot be optimized. The canonical example where optimization is successful is the case where the bispectrum is given exactly by an integral over conformal distance $r$ as in Eq. (9). In this case, the input bispectrum (Eq. (31)) could be obtained by oversampling the integral using a large number $N_{\text {fact }}$ of quadrature points in $r$. The output bispectrum (Eq. (32)) would represent a more efficient quadrature, specifically tailored to the $r$ dependence of the bispectrum under consideration, using a smaller number $N_{\text {opt }}$ of points, with integration weights given by the $w_{i}$.

For Fisher matrix forecasts, it is often unnecessary to optimize $N_{\text {fact }}$; as discussed in $₫ \mathrm{IV}$, the Fisher matrix can frequently be computed in harmonic space even if the number of factorizable terms is large. However, for the analysis and simulation algorithms which will be presented in $\mathrm{VII}$ IX we will see that optimizing $N_{\text {fact }}$ as a preliminary step usually makes a large improvement in the cost. This is the main benefit of the optimization algorithm which we now discuss.

\section{A. Optimization algorithm}

Let us first ask: in what sense is one bispectrum $B_{\ell_{1} \ell_{2} \ell_{3}}^{\prime}$ a good approximation for another bispectrum $B_{\ell_{1} \ell_{2} \ell_{3}}$ ? Our criterion is based on distinguishability; the approximation is good if the Fisher distance

$$
F\left(B, B^{\prime}\right) \stackrel{\text { def }}{=} \frac{1}{6} \sum_{\ell_{1} \ell_{2} \ell_{3}} \frac{\left(B_{\ell_{1} \ell_{2} \ell_{3}}-B_{\ell_{1} \ell_{2} \ell_{3}}^{\prime}\right)^{2}}{C_{\ell_{1}} C_{\ell_{2}} C_{\ell_{3}}}
$$

is small. Here, $C_{\ell}$ is a signal + noise power spectrum characterizing the survey under consideration, which is required as an input to our optimization algorithm. We usually iterate our algorithm until $F\left(B, B^{\prime}\right)$ is of order $10^{-6}$ or smaller; this corresponds to an optimized bispectrum which cannot be distinguished from the original to better than $0.001 \sigma$. (In a realistic survey, the noise will be inhomogeneous and hence not describable by a power spectrum, but because our termination criterion is so conservative, it suffices to use a noise power spectrum which roughly models the survey.)

As a first step toward an optimization algorithm, suppose that we have already chosen a subset of terms to retain, and want to choose optimal (in the sense that the Fisher distance $F\left(B, B^{\prime}\right)$ is minimized) values for the weights $w_{i}$. In $\llbracket$ IV we showed (Eq. (29) how to efficiently calculate the $N_{\text {fact }}$-by- $N_{\text {fact }}$ Fisher matrix $F_{i j}$ between the individual terms in the input bispectrum (Eq. (31)). If $F$ is block decomposed into submatrices of size $N_{\text {opt }}$ and $\left(N_{\text {fact }}-N_{\text {opt }}\right)$,

$$
F=\left(\begin{array}{ll}
F_{00} & F_{01} \\
F_{01}^{T} & F_{11}
\end{array}\right)
$$

then the Fisher distance is given by

$$
\begin{aligned}
F\left(B, B^{\prime}\right)=\sum_{i j} & \left(1-w_{i}\right)\left(F_{00}\right)_{i j}\left(1-w_{j}\right) \\
& +2 \sum_{i J}\left(1-w_{i}\right)\left(F_{01}\right)_{i J}+\sum_{I J}\left(F_{11}\right)_{I J}
\end{aligned}
$$

Note that we use lower case to denote indices in the first blocks of the decomposition in Eq. (34) and upper case for the second block. The Fisher distance $F\left(B, B^{\prime}\right)$ is minimized by choosing

$$
\left(w_{i}\right)_{\mathrm{opt}}=1+\sum_{J}\left(F_{00}^{-1} F_{01}\right)_{i J}
$$


and the value at the minimum is given by

$$
F\left(B, B^{\prime}\right)_{\mathrm{opt}}=\sum_{I J}\left(F_{11}-F_{01}^{T} F_{00}^{-1} F_{01}\right)_{I J}
$$

Now that we have seen how to optimize the weights, we address the problem of choosing which terms in the original bispectrum (Eq. (31)) to retain. Mathematically, this corresponds to choosing a subset of terms such that $F\left(B, B^{\prime}\right)_{\text {opt }}$ (Eq. (37) ) is minimized. (Note that after setting the $w_{i}$ to their optimal values, $F\left(B, B^{\prime}\right)$ opt still depends on the subset of terms which are retained, but this dependence is hidden in Eq. (37), which follows our convention of assuming that the terms have been permuted so that terms $\left\{1, \ldots, N_{\text {opt }}\right\}$ are retained.) Since exhaustive search of all subsets would be prohibitively slow, our approach is to use a greedy algorithm: we build up a subset of terms iteratively, in each iteration adding the term which results in the greatest improvement in $F\left(B, B^{\prime}\right)_{\mathrm{opt}}$. The algorithm terminates when $F\left(B, B^{\prime}\right)$ opt has reached an acceptably small value.

The simplest implementation of this algorithm would evaluate $F\left(B, B^{\prime}\right)$ opt from scratch, using Eq. (37), for each candidate term in each iteration, which would lead to a running time of $\mathcal{O}\left(N_{\mathrm{opt}}^{2} N_{\text {fact }}^{3}\right)$, in addition to the time needed to precompute the matrix $F_{i j}$. This can be improved by two optimizations. First, in the $n$-th iteration of the algorithm, suppose we have chosen $n$ terms to retain and want to choose the $(n+1)$-st. If the matrix $F$ is decomposed into blocks of size $n$ and $\left(N_{\text {fact }}-n\right)$ as in Eq. (34), then one can show that the change in $F\left(B, B^{\prime}\right)_{\text {opt }}$ if the $I$-th term is added is given by:

$$
\Delta F\left(B, B^{\prime}\right)_{\mathrm{opt}}=\frac{\left(\sum_{J}\left(F_{11}-F_{01}^{T} F_{00}^{-1} F_{01}\right)_{I J}\right)^{2}}{\left(F_{11}-F_{01}^{T} F_{00}^{-1} F_{01}\right)_{I I}}
$$

After computing the matrix $\left(F_{11}-F_{01}^{T} F_{00}^{-1} F_{01}\right)$, Eq. (38) allows us to select the $(n+1)$-st term to be retained in time $\mathcal{O}\left(N_{\text {fact }}^{2}\right)$. This optimization improves the cost from $\mathcal{O}\left(N_{\text {opt }}^{2} N_{\text {fact }}^{3}\right)$ to $\mathcal{O}\left(N_{\text {opt }} N_{\text {fact }}^{3}\right)$; the limiting step is recomputing the matrix $\left(F_{11}-F_{01}^{T} F_{00}^{-1} F_{01}\right)$ from scratch for each $n$.

This brings us to the second optimization: instead of keeping the matrix $F$, in each iteration we keep the matrix $G$ defined by:

$$
G \stackrel{\text { def }}{=}\left(\begin{array}{cc}
-F_{00}^{-1} & -F_{00}^{-1} F_{01} \\
-F_{01}^{T} F_{00}^{-1} & F_{11}-F_{01}^{T} F_{00}^{-1} F_{01}
\end{array}\right)
$$

Note that the lower right block of $G$ is the matrix needed to efficiently select the next term, as described in the previous paragraph. The other blocks have been constructed so that it is possible to update $G$ in $\mathcal{O}\left(N_{\text {fact }}^{2}\right)$ time when advancing from the $n$-th iteration of the algorithm to the $(n+1)$-st iteration (rather than recomputing from scratch at cost $\left.\mathcal{O}\left(N_{\text {fact }}^{3}\right)\right)$. More precisely, assuming that terms have been permuted so that the new term to be retained is in the $(n+1)$-st position, the update rule is given as follows. If $G$ is given by the block decomposition (into sizes $n$ and $\left.\left(N_{\text {fact }}-n\right)\right)$

$$
G=\left(\begin{array}{cc}
G_{00} & G_{01} \\
G_{01}^{T} & G_{11}
\end{array}\right)=\left(\begin{array}{c|cc}
G_{00} & v_{0} & A_{0} \\
\hline v_{0}^{T} & \gamma & v_{1}^{T} \\
A_{0}^{T} & v_{1} & A_{1}
\end{array}\right)
$$

in the $n$-th iteration, then it is given by

$$
G \rightarrow\left(\begin{array}{cc|c}
G_{00}-(1 / \gamma) v_{0} v_{0}^{T} & -v_{0} / \gamma & A_{0}-(1 / \gamma) v_{0} v_{1}^{T} \\
-v_{0}^{T} / \gamma & -1 / \gamma & -v_{1}^{T} / \gamma \\
\hline A_{0}^{T}-(1 / \gamma) v_{1} v_{0}^{T} & -v_{1} / \gamma & A_{1}-(1 / \gamma) v_{1} v_{1}^{T}
\end{array}\right)
$$

in the $(n+1)$-st iteration. (Note that the middle "block" in Eqs. (40), (41) has size 1, e.g. $\gamma$ is a number.) By keeping the matrix $G$, and using the update rule (Eq. (41)), the cost of the optimization algorithm is further improved to $\mathcal{O}\left(N_{\text {opt }} N_{\text {fact }}^{2}\right)$.

Putting this all together, our optimization algorithm can be summarized as follows. We initialize the $G$-matrix to the matrix $F_{i j}$ defined in Eq. (29), and initialize the "score" $F\left(B, B^{\prime}\right)$ to the sum of the entries of $F$. We then iterate the following loop (starting with $n=0$ ):

1. In the $n$-th iteration, we have already chosen a subset of $n$ terms to retain and permuted the original terms so that these are in positions $1, \ldots, \mathrm{n}$. We have stored the $G$-matrix defined by the block decomposition in Eq. (40).

2. Choose the index $I=n+1, \ldots, N_{\text {fact }}$ which maximizes

$$
-\Delta F\left(B, B^{\prime}\right)_{\mathrm{opt}}=\frac{\left(\sum_{J} G_{I J}\right)^{2}}{G_{I I}}
$$

This represents the $(n+1)$-st term chosen to be retained.

3. Update $F\left(B, B^{\prime}\right)_{\text {opt }}$ according to Eq. (38), permute the terms so that the new term is in the $(n+1)$-st position, and update the $G$-matrix according to the rule in Eq. (41).

4. If $F\left(B, B^{\prime}\right)$ has become acceptably small, then terminate, returning the subset of terms to be retained and the optimal weights

$$
\left(w_{i}\right)_{\mathrm{opt}}=1-\sum_{J} G_{i J}
$$

Otherwise, continue to the $(n+1)$-st iteration.

The total running time is $\mathcal{O}\left(N_{\text {opt }} N_{\text {fact }}^{2}\right)$, which in practice is usually less than the $\mathcal{O}\left(\ell_{\max }^{2} N_{\text {fact }}^{2}\right)$ time needed to precompute the matrix $F_{i j}$. 


\begin{tabular}{|c|c|c|c|c|c|}
\hline & $\nu$ & $\theta_{\text {FWHM }}$ & $\Delta_{T}$ & $f_{\text {sky }}$ & $\ell_{\text {max }}$ \\
\hline WMAP3 & $41 \mathrm{GHz}$ & $28^{\prime}$ & $460 \mu \mathrm{K}^{\prime}$ & 0.77 & 1000 \\
& $61 \mathrm{GHz}$ & $20^{\prime}$ & $560 \mu \mathrm{K}^{\prime}$ & 0.77 & 1000 \\
& $94 \mathrm{GHz}$ & $12^{\prime}$ & $680 \mu \mathrm{K}^{\prime}$ & 0.77 & 1000 \\
\hline Planck & $100 \mathrm{GHz}$ & $9.2^{\prime}$ & $51 \mu \mathrm{K}^{\prime}$ & 0.8 & 2000 \\
& $143 \mathrm{GHz}$ & $7.1^{\prime}$ & $43 \mu \mathrm{K}^{\prime}$ & 0.8 & 2000 \\
& $217 \mathrm{GHz}$ & $5.0^{\prime}$ & $65 \mu \mathrm{K}^{\prime}$ & 0.8 & 2000 \\
\hline
\end{tabular}

TABLE I: Experimental parameters used when making Fisher forecasts for three-year WMAP and Planck, taken from the WMAP3 public data release and [4] respectively.

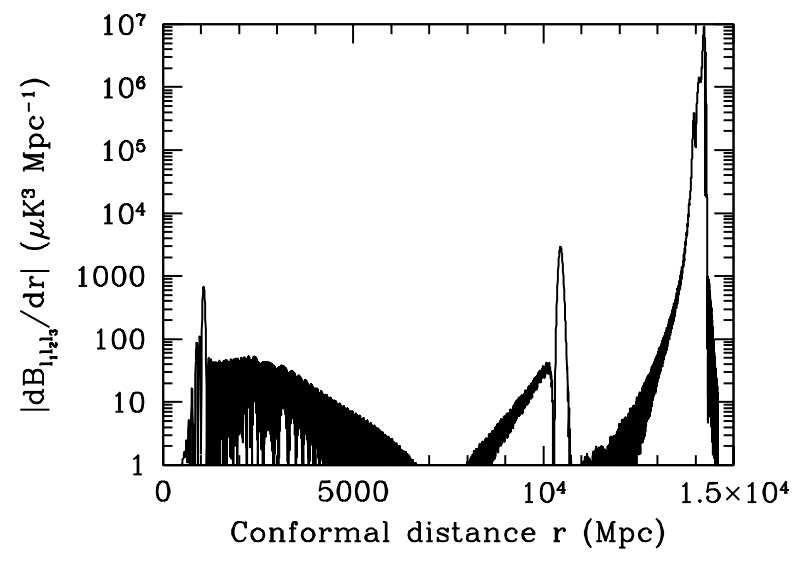

FIG. 1: Contribution to $B_{\ell_{1} \ell_{2} \ell_{3}}^{\text {loc }}$ as a function of conformal distance $r$, with dominant contribution from recombination $(r \sim 14000 \mathrm{Mpc})$, for $\left(\ell_{1}, \ell_{2} \ell_{3}\right)=(2,300,300)$, a typical squeezed triple with high signal-to-noise.

\section{B. Optimization examples}

We now give some examples of our algorithm for optimizing $N_{\text {fact }}$, using bispectra from $₫$ III. We use noise power spectra which roughly approximate the three-year WMAP and Planck surveys, as given in Tab. I) (Note that, because the optimization algorithm is Fisher distance based, a noise power spectrum is one of the inputs.)

First consider the local bispectrum $b_{\ell_{1} \ell_{2} \ell_{3}}^{\text {loc }}$, which is an integral over conformal distance $r$ (Eq. (13)), with one factorizable term for each quadrature point needed to do the integral. To get a feeling for how different values of $r$ contribute, we show the integrand for $\left(\ell_{1}, \ell_{2}, \ell_{3}\right)=(2,300,300)$ in Fig. 1. (This particular triple was selected for high signal-to-noise; the local bispectrum is dominated by "squeezed" triples with $\ell_{1} \ll \ell_{2}, \ell_{3}$.) This structure, showing a large contribution from recombination $(r \sim 14000 \mathrm{Mpc})$, with secondary contributions from reionization $(r \sim 10500)$ and ISW $(r \lesssim 5000)$, is typical of bispectra which arise from primordial nonGaussianity.

To be conservative, we oversample the $r$ integral using a quadrature with 1200 points as shown in Tab. II

\begin{tabular}{|c|c|}
\hline $0 \leq r \leq 9500$ & $N_{\text {quad }}=150$, linearly-spaced \\
$9500 \leq r \leq 11000$ & $N_{\text {quad }}=300$, linearly-spaced \\
$11000 \leq r \leq 13800$ & $N_{\text {quad }}=150$, linearly-spaced \\
$13800 \leq r \leq 14600$ & $N_{\text {quad }}=400$, linearly-spaced \\
$14600 \leq r \leq 16000$ & $N_{\text {quad }}=100$, linearly-spaced \\
$16000 \leq r \leq 50000$ & $N_{\text {quad }}=100$, log-spaced \\
\hline
\end{tabular}

TABLE II: Quadrature in $r$ used when computing bispectra in $\mathrm{VB}$, with a greater density of points near reionization (second row) and recombination (fourth row); units for $r$ are Mpc.

\begin{tabular}{|c||c|c|c|}
\hline & $\begin{array}{c}N_{\text {fact }} \\
\text { (input) }\end{array}$ & $\begin{array}{c}N_{\text {opt }} \\
\text { (WMAP3) }\end{array}$ & $\begin{array}{c}N_{\text {opt }} \\
\text { (Planck) }\end{array}$ \\
\hline Point source (Eq. (7) $)$ & 1 & 1 & 1 \\
ISW-lensing (Eq. (44) $)$ & 3 & 3 & 3 \\
\hline Local (Eq. (12)) & 1200 & 11 & 21 \\
Equilateral (Eq. (22)) & 3600 & 24 & 47 \\
Gravitational (Eq. (16) $)$ & 4800 & 168 & 255 \\
HD (Eq. (19) & 80000 & 33 & 86 \\
\hline
\end{tabular}

TABLE III: Number of terms $N_{\text {fact }}$ obtained for the point source, ISW-lensing, local, equilateral, gravitational, and higher derivative bispectra, after oversampling the integrals using the integration quadratures described in $\mathrm{VB}$, and number of terms $N_{\text {opt }}$ which are retained after using the optimization algorithm for WMAP3 and Planck noise levels.

This quadrature was obtained empirically by increasing the sampling until each of the bispectra considered in §III had converged at the percent level, for several representative choices of $\left(\ell_{1}, \ell_{2}, \ell_{3}\right)$. Using this quadrature for the local bispectrum, we obtain a bispectrum with $N_{\text {fact }}=1200$. After running the optimization algorithm, optimized bispectra with $N_{\text {opt }}=11$ or 21 factorizable terms are obtained, for WMAP3 or Planck noise levels respectively. In this case, the optimization algorithm can be thought of as computing a more efficient quadrature in $r$ by choosing both quadrature points and integration weights $w_{i}$. The resulting quadrature is optimized so that it results in a bispectrum which is indistinguishable at the given noise levels from the oversampled bispectrum, while using far fewer quadrature points.

Repeating this procedure for the gravitational (Eq. (16)) and equilateral (Eq. (22)) bispectra, we arrive at the $N_{\text {opt }}$ values given in the middle rows of Tab. III. We emphasize that in all cases, the input and output bispectra are instinguishable (to $0.001 \sigma$ ) at the given sensitivity levels, since the optimization algorithm is only allowed to terminate when the Fisher distance $F\left(B_{\text {input }}, B_{\text {opt }}\right)$ is $\leq 10^{-6}$.

As a final example, consider the higher-derivative bispectrum (Eq. (20)). In this case, the bispectrum is a double integral over conformal distance $r$ and Schwinger parameter $t$. For the $t$ integral, we use a uniform quadra- 


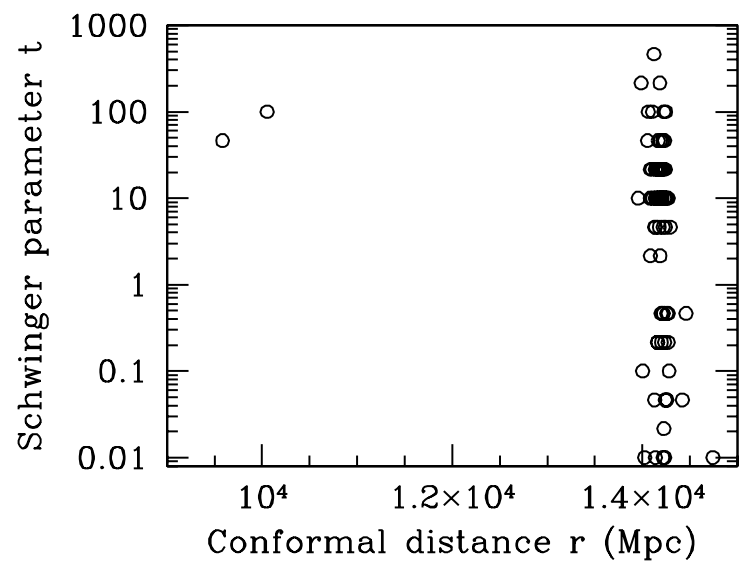

FIG. 2: Distribution of factorizable terms in the $(r, t)$ plane for the "optimized" higher-derivative bispectrum $b_{\ell_{1} \ell_{2} \ell_{3}}^{\text {hd }}$.

ture in $\log (t)$ with three points per decade from $t=10^{-2}$ to $t=10^{6}$. With this quadrature, we find that the identity

$$
\frac{1}{\left(k_{1}+k_{2}+k_{3}\right)^{2}}=\int_{0}^{\infty} d t t e^{-t\left(k_{1}+k_{2}+k_{3}\right)}
$$

holds to $0.1 \%$, throughout the range of wavenumbers $10^{-6} \lesssim k \lesssim 1 \mathrm{Mpc}^{-1}$ where the photon transfer function $\Delta_{\ell}(k)$ is appreciably different from zero (for $\ell_{\max }=$ 2000). As explained in 乌III Eq. (44) is the basis for writing the higher-derivative bispectrum in factorizable form. For the $r$ integral, we use the quadrature in Tab. II, with one additional sublety mentioned for completeness: at large values of $t$, we have found that the inner integral over $r$ contains contributions from large values of $r$. For this reason, we extend the log-spaced quadrature in the last row of Tab. II to $r_{\max }=\left(5 \times 10^{6}\right)$ using 400 additional points.

Combining this 25-point quadrature in $t$ and 1600point quadrature in $r$, and with 2 factorizable terms per quadrature point, we obtain an "oversampled" higherderivative bispectrum with $N_{\text {fact }}=80000$. It is infeasible to optimize this bispectrum directly since the cost of the optimization algorithm is $\mathcal{O}\left(N_{\text {fact }}^{2} \ell_{\text {max }}^{2}\right)$. For this reason, we use a two-level optimization procedure, first separating the factorizable terms into batches with $\sim 1000$ terms which are optimized separately, then combining the "optimized batches" into one bispectrum which is optimized again. The final result is a bispectrum with $N_{\text {fact }}=33$ or 86 , for WMAP3 or Planck respectively. For Planck, we show the distribution of terms in the $(r, t)$ plane in Fig. 2. Even though the oversampled bispectrum contains terms throughout the ranges $10^{-2} \leq t \leq 10^{6}$ and $0 \leq r \leq\left(5 \times 10^{6}\right)$, the optimization algorithm finds that a much smaller range in $(r, t)$ suffices to accurately approximate the bispectum.

In more detail, the two-level optimization procedure used for the higher-derivative bispectrum is given as fol- lows. The input bispectrum is split into $N$ bispectra $\left\{B_{\text {input }}^{(i)}\right\}$ :

$$
B_{\text {input }}=\sum_{i=1}^{N} B_{\text {input }}^{(i)}
$$

each of which is optimized separately, obtaining bispectra $\left\{B_{\mathrm{opt}}^{(i)}\right\}$ which satisfy:

$$
F\left(B_{\text {input }}^{(i)}, B_{\text {opt }}^{(i)}\right) \leq \frac{10^{-6}}{4 N^{2}}
$$

These are combined for a final round of the optimization algorithm, obtaining an output bispectrum $B_{\text {opt }}$ which satisfies:

$$
F\left(B_{\mathrm{opt}}, \sum_{i} B_{\mathrm{opt}}^{(i)}\right) \leq \frac{10^{-6}}{4}
$$

With the threshhold values given on the right-hand sides of Eqs. (46), (47), it can be proved that the output bispectrum satisfies $F\left(B_{\text {input }}, B_{\text {output }}\right) \leq 10^{-6}$, our standard termination criterion. Thus the accuracy of the approximation need not be degraded by use of the two-level procedure.

An interesting and counterintuitive byproduct of the two-level procedure is that, even when the number of terms in $B_{\text {input }}$ is so large that direct computation of the 1-by-1 Fisher matrix $F\left(B_{\text {input }}, B_{\text {input }}\right)$ is infeasible, it may be possible to obtain an optimized bispectrum $B_{\text {opt }}$ whose Fisher distance to $B_{\text {input }}$ is provably small. This increases the scope of the factorizability ansatz: even if the number of terms required to represent a bispectrum in factorizable form appears to be intractably large, the optimization procedure may succeed in reducing to a more efficient representation, for which the algorithms described in this paper will be affordable.

Let us conclude by emphasizing the sense in which the output bispectrum from the optimization algorithm approximates the input bispectrum. The only requirement is that the two are experimentally indistinguishable to $0.001 \sigma$. Intuitively, this means that they approximate each other at percent level in regimes which contribute the most signal-to-noise. In regimes where the input bispectrum is too small to contribute significant signal-tonoise, the output bispectrum is not guaranteed to resemble the input; it is only guaranteed also to be small. This is why, for example, our optimization algorithm tends to drop all contributions after reionization $(r \lesssim 9500)$; these mainly contribute (via the ISW effect) to triples $\left(\ell_{1}, \ell_{2}, \ell_{3}\right)$ in which each $\ell_{i}$ is small, and the total contribution of such triples to the Fisher matrix is negligible. (In fact, such contributions could presumably be dropped from the outset, but our approach is to conservatively oversample the integrals and let the optimization algorithm determine which contributions are negligible.)

Our focus will be on Fisher matrix forecasts and bispectrum estimation, for which this distinguishabilitybased criterion is ideal, since it permits extremely aggressive optimization of the bispectrum, as seen in Tab. III. 


\begin{tabular}{|c|cccccc|}
\hline & Pt. src. & ISW & Loc. & Eq. & Grav. & HD \\
\hline Pt. src. & $(0.05)$ & 0.00 & 0.00 & -0.01 & 0.00 & -0.01 \\
ISW & 0.00 & $(0.16)$ & -0.24 & 0.00 & 0.25 & 0.01 \\
Local & 0.00 & -0.24 & $(6.3)$ & 0.25 & 0.78 & 0.28 \\
Equil. & -0.01 & 0.00 & 0.25 & $(66.9)$ & 0.36 & -0.98 \\
Grav. & 0.00 & 0.25 & 0.77 & 0.36 & $(33.4)$ & 0.28 \\
HD & -0.01 & 0.01 & 0.28 & -0.98 & 0.28 & $(59.9)$ \\
\hline
\end{tabular}

TABLE IV: Fisher matrix for Planck, using bispectra from Tab. III and noise parameters from Tab. I] Off-diagonal entries are correlations; the diagonal parenthesized entries are $1 \sigma$ Fisher matrix errors $\left(=F_{i i}^{-1 / 2}\right)$ on the amplitude of each bispectrum, without marginalizing the others.

However, the optimization algorithm is not without caveats. As mentioned above, the optimization algorithm is allowed to alter the shape of the bispectrum for triples $\left(\ell_{1}, \ell_{2}, \ell_{3}\right)$ in which the bispectrum is so small that it makes a negligible contribution to the Fisher matrix. One could worry that this could make the optimized bispectrum more sensitive to systematic errors, if there is some observational reason why these triples are sensitive to systematic effects not modeled by the Fisher formalism. If this is a concern, then a direct test for systematic contamination can be performed, by verifying that the optimized and unoptimized shapes give nearly identical values when "contracted" with real data (i.e. that the values of $T\left[C^{-1} a\right]$ are nearly identical, in notation from §VII). Another concern is that "closeness" of the optimized and unoptimized shapes, in the Fisher matrix sense, does not strictly guarantee that the sampling PDFs of the corresponding three-point estimators must be nearly identical (although it does guarantee that the two PDFs have variances that are nearly equal). We verified directly that this is not a concern for the local and equilateral shapes, but defer a more systematic study of this question for future work.

\section{FORECASTS FOR PLANCK}

Armed with the optimized bispectra in Tab. III it is straightforward to perform a Fisher matrix analysis for Planck, using noise parameters from Tab. I. The result is shown in Tab. IV] we obtain $\sigma\left(f_{N L}^{\text {loc }}\right)=6.3$, $\sigma\left(f_{N L}^{\mathrm{eq}}\right)=66.9$ and a $6 \sigma$ detection (corresponding to $1 \sigma$ error 0.16 ) of the ISW-lensing bispectrum. For the point source bispectrum, we have taken a reference value $b^{\mathrm{ps}}=10^{-8} \mu \mathrm{K}^{3}$ as described in $₫ \mathrm{III}$, and found $\mathrm{a} \sim 20 \sigma$ detection, but this figure should be taken very roughly since the value of $b^{\mathrm{ps}}$ is very sensitive to the flux limit which is assumed.

One result from the Fisher matrix forecast is that the higher-derivative bispectrum (Eq. (19)) is $98 \%$ correlated to the equilateral shape (Eq. (22)). In practice, this means that it suffices to use the (simpler) equilateral form

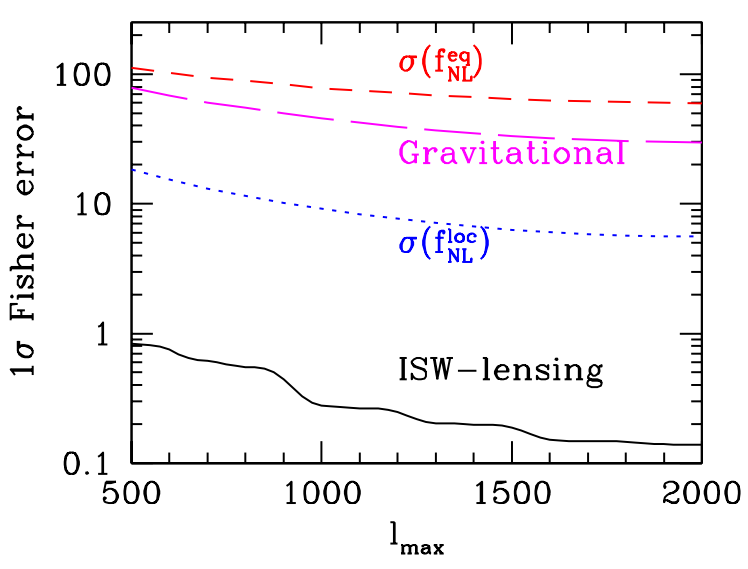

FIG. 3: Fisher matrix errors vs. $\ell_{\max }$ for the ISW-lensing, local, equilateral, and gravitational forms of the bispectrum, assuming Planck noise levels throughout.

when analyzing data; the two bispectra cannot be distinguished (at $1 \sigma$ ) unless a $25 \sigma$ detection can also be made. This result agrees well with [19], where a 0.98 correlation was also found.

A second result is that the gravitational bispectrum (Eq. (16)) is well below the detectability threshhold for Planck. (We also find that it is $97 \%$ correlated to a linear combination of the local and equilateral bispectra, but this is not relevant if its amplitude is too small to be detected.) One way to quantify this statement is by quoting an "effective" value of $f_{N L}$ for which the local bispectrum (Eq. (12)) has the same Fisher matrix error as the gravitational bispectrum (whose amplitude is assumed fixed). Using the Fisher matrix we have computed, we obtain $f_{N L}^{\text {eff }}=0.2$. This value does not depend strongly on $\ell_{\max }$, as can be seen in Fig. 3, where we show the dependence of the Fisher matrix errors on $\ell_{\max }$, assuming Planck noise characteristics throughout.

This result seems to disagree with [41], who found $f_{N L}^{\mathrm{eff}} \approx 4$ at $\ell_{\max }=500$ with a trend toward increasing $f_{N L}^{\text {eff }}$ as $\ell_{\max }$ increases. Since the two methods for calculating the Fisher matrix are so different, it is difficult to compare the calculations directly. However, the result that the gravitational bispectrum is "weaker" than the local bispectrum with $f_{N L}^{\text {loc }}=1$ can be seen intuitively from the 3D bispectra in Eqs. (12), (16). One can prove that the ratio of the two bispectra satisfies

$$
0 \leq\left|\frac{F^{\text {grav }}\left(k_{1}, k_{2}, k_{3}\right)}{F^{\text {loc }}\left(k_{1}, k_{2}, k_{3}\right)}\right| \leq \frac{13}{12}
$$

for all values of $\left(k_{1}, k_{2}, k_{3}\right)$ which satisfy the triangle inequality, and the ratio is close to zero for the "squeezed" configurations which contribute greatest signal-to-noise. For example, in the squeezed limit $k_{2}=k_{3}, k_{1} \rightarrow 0$, the ratio approaches $1 / 6$.

Another conclusion from the Fisher matrix forecast is that the equilateral bispectrum seems more difficult to 

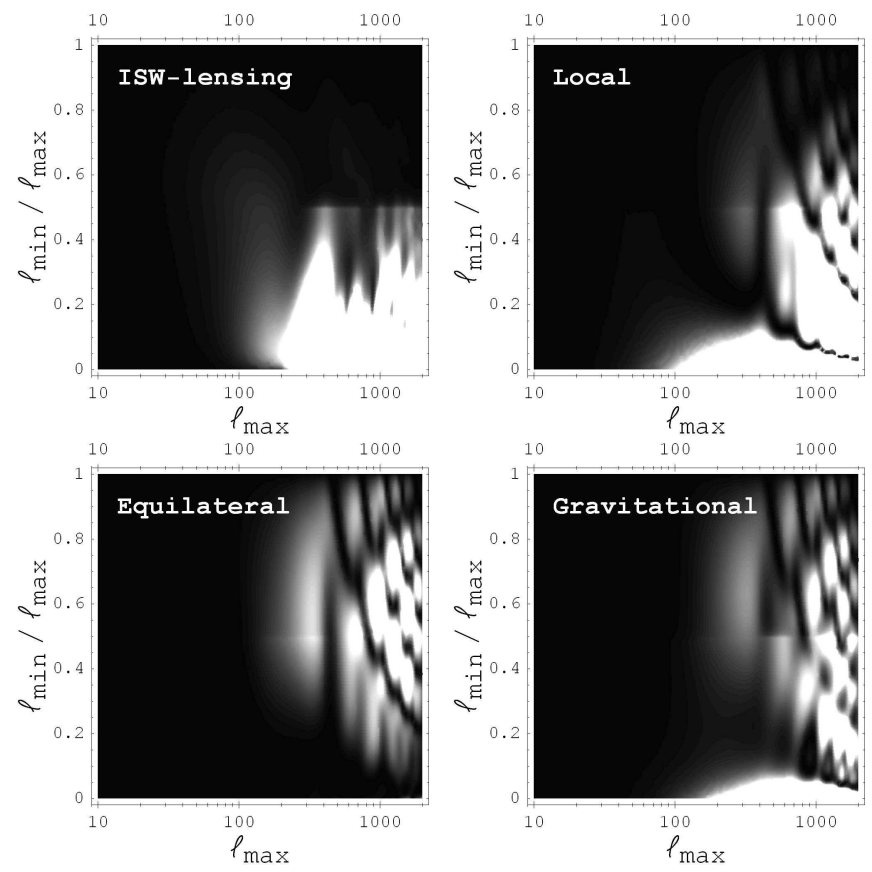

FIG. 4: Contour plots of $d F / d\left(\log \ell_{\max }\right) d\left(\ell_{\min } / \ell_{\max }\right)$, defined in Eq. (49), showing the contribution to the Fisher matrix error as a function of $\left(\ell_{\min }, \ell_{\max }\right)$, for the ISW-lensing, local, equilateral, and gravitational forms of the bispectrum.

detect than the local bispectrum. This is in some sense just a matter of convention in the definition $f_{N L}$ in the different cases. This has already been observed in the context of WMAP; e.g. in [44], the $1 \sigma$ errors $\sigma\left(f_{N L}^{\text {loc }}\right)=34$ and $\sigma\left(f_{N L}^{\mathrm{eq}}\right)=147$ were obtained from three-year WMAP data. Here we find (Fig. (3) that this trend becomes somewhat more pronounced with increasing $\ell_{\max }$; for Planck $\left(\ell_{\max }=2000\right)$, the ratio $\sigma\left(f_{N L}^{\mathrm{eq}}\right) / \sigma\left(f_{N L}^{\mathrm{loc}}\right)$ has increased to 10.6 .

Finally, we find that correlations between the point source, ISW-lensing, local, and equilateral bispectra are small. In effect, the CMB gives independent constraints on these four bispectra which are not appreciably degraded by marginalizing the other three. However, the correlation between the ISW-lensing and local bispectra is large enough that the presence of the former (a guaranteed $6 \sigma$ signal) contributes non-negigible bias $\left(\Delta f_{N L}^{\text {loc }}=9.8\right)$ when estimating the latter. If a multibispectrum analysis with marginalization is performed, then this bias will be subtracted without significantly degrading $\sigma\left(f_{N L}^{\text {loc }}\right)$. A similar comment applies to the point source bispectrum; we have found that at the Planck reference value $\left(b^{\mathrm{ps}}=10^{-8} \mu \mathrm{K}^{3}\right)$, there is negligible bias contributed to the other bispectra, but the point source bispectrum should be marginalized in practice since its value is quite uncertain.

It is illuminating to show the contributions to the Fisher matrix from differently shaped triples $\left(\ell_{1}, \ell_{2}, \ell_{3}\right)$.

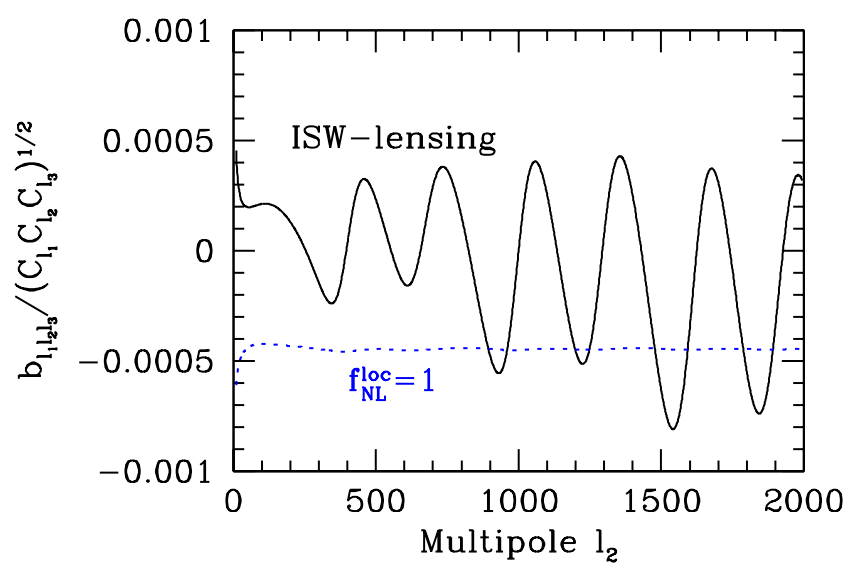

FIG. 5: Values of $b_{\ell_{1}, \ell_{2} \ell_{3}} /\left(C_{\ell_{1}} C_{\ell_{2}} C_{\ell_{3}}\right)^{1 / 2}$, showing oscillations in the ISW-lensing bispectrum but not the local bispectrum, plotted for $\ell_{1}=10$ and $\ell_{3}=\ell_{2}+6$. This is a typical "squeezed" triangle which contributes high signal-to-noise in both cases.

In Fig. 4 , we show contour plots of the quantity

$$
\frac{d F}{d \log \left(\ell_{\max }\right) d\left(\ell_{\min } / \ell_{\max }\right)}=\ell_{\max }^{2} \sum_{\ell=\ell_{\min }}^{\ell_{\max }} \frac{\left(B_{\ell_{\min }, \ell, \ell_{\max }}\right)^{2}}{6 C_{\ell_{\min }} C_{\ell} C_{\ell_{\max }}}
$$

The Jacobian $\left(\ell_{\max }^{2}\right)$ has been included as a prefactor so that the Fisher matrix element $F$ will be given by integrating over the variables $\left\{\log \left(\ell_{\max }\right),\left(\ell_{\min } / \ell_{\max }\right)\right\}$ on the axes of the plot. The sharp feature seen in these plots at $\ell_{\min } / \ell_{\max }=(1 / 2)$ arises solely from the behavior of the Wigner $3 \mathrm{j}$ symbols, and would be present even if the reduced bispectrum $b_{\ell_{1} \ell_{2} \ell_{3}}$ were simply proportional to $\left(C_{\ell_{1}} C_{\ell_{2}} C_{\ell_{3}}\right)^{1 / 2}$.

It is seen that the equilateral bispectrum receives most of its signal-to-noise from the "equilateral" regime $\left(\ell_{\min }\right.$ comparable to $\ell_{\max }$ ), whereas the ISW-lensing and local bispectra receive highest contributions from the squeezed regime $\left(\ell_{\min } \ll \ell_{\max }\right)$, confirming the intuitive picture from the end of $\oiiint$ III From this description it may seem surprising that the correlation between the ISW-lensing and local bispectra is so small (0.25). This is because the ISW-lensing bispectrum contains oscillatory features which are not present in the local case (Fig. (5)). These are hidden in Fig. 4 and help orthogonalize the two bispectra. If one were to artificially replace $b_{\ell_{1} \ell_{2} \ell_{3}} \rightarrow\left|b_{\ell_{1} \ell_{2} \ell_{3}}\right|$, then the correlation between the ISW-lensing and local bispectra would increase to 0.6 .

\section{OPTIMAL BISPECTRUM ESTIMATION}

In this section, we present a general framework for optimal bispectrum estimation in the presence of sky cuts and inhomogeneous noise. In the context of power spectrum estimation, a method similar to ours was proposed 
by $\mathrm{Oh}$ et al. [22], and it is illuminating to first revisit their construction.

\section{A. Power spectrum estimation}

The power spectrum estimation problem can be stated as follows. One is interested in simultaneously estimating the amplitude of bandpowers $C_{1}, \ldots, C_{N_{\text {band }}}$ from a map $m$ with noise covariance $N$. It can be shown [20] that the optimal estimator $\mathcal{E}_{\alpha}$ for each bandpower $\alpha$ is given by

$$
\mathcal{E}_{\alpha}[m] \stackrel{\text { def }}{=} \frac{1}{2} F_{\alpha \beta}^{-1}\left(m^{T} C^{-1} C_{\beta} C^{-1} m\right),
$$

where $C=S+N$ is the total signal + noise, $m$ is the observed temperature map (assumed to have covariance $C$ ), and $F_{\alpha \beta}$ is the Fisher matrix

$$
F_{\alpha \beta} \stackrel{\text { def }}{=} \frac{1}{2} \operatorname{Tr}\left[C_{\alpha} C^{-1} C_{\beta} C^{-1}\right] .
$$

A subtlety in Eqs. (50), (51) is that, strictly speaking, the definition of the estimator depends (via the matrix $C^{-1}$ ) on the signal covariance $S$ which one is trying to estimate in the first place. In practice, the estimator can be iterated until convergence is reached; it can be shown that the limiting value of $S$ obtained in this way is equal to a maximum likelihood estimate [20]. This subtlety will be ignored in this section where our purpose is merely to set the stage, in the more familiar context of power spectrum estimation, for the bispectrum estimator which follows.

Evaluating Eqs. (50), (51) appears infeasible for large maps owing to the $\mathcal{O}\left(N_{\text {pix }}^{3}\right)$ matrix operations which appear. However, the computational cost can be reduced by avoiding use of dense matrices (e.g. [22, 45]). Considering Eq. (50) first, one only needs to multiply a single map by $C^{-1}$, which can often be done affordably (and without needing to store the matrix $C$ in dense form) using conjugate gradient inversion, although the details will depend on the experiment's noise model. Considering next Eq. (51), the trace can be written as as a Monte Carlo average:

$$
F_{\alpha \beta}=\frac{1}{2}\left\langle a^{T} C^{-1} C_{\alpha} C^{-1} C_{\beta} C^{-1} a\right\rangle_{a},
$$

where the notation $\langle\cdot\rangle_{a}$ denotes an average taken over signal + noise realizations $a$ (i.e., $a$ is a Gaussian random field with covariance $C$ ). If we continue to assume an affordable procedure for multipliying a map by $C^{-1}$, Eq. (52) permits $F_{\alpha \beta}$ to be computed by Monte Carlo. The estimator covariance is then given by

$$
\operatorname{Cov}\left(\mathcal{E}_{\alpha}, \mathcal{E}_{\beta}\right)=F_{\alpha \beta}^{-1} \text {. }
$$

The matrix $F_{\alpha \beta}$, defined by Eq. (51), is the Fisher matrix for the survey with noise covariance given by an arbitrary matrix $N$. For optimal estimators, the Fisher matrix gives both the normalization (Eq. (50)) and the covariance (Eq. (53)).

This method for optimal power spectrum estimation in the presence of arbitrary noise covariance $C$ has an analogue for bispectra, as we will now see.

\section{B. Bispectrum estimation}

Let us now consider the analagous problem of optimal estimation of the amplitude of a given bispectrum $B_{\ell_{1} \ell_{2} \ell_{3}}$. The form of the optimal estimator has been constructed previously [46] and shown to contain both cubic and linear terms:

$$
\begin{aligned}
& \mathcal{E}[a]=\frac{1}{6 F_{\mathcal{E}}} B_{\ell_{1} \ell_{2} \ell_{3}}\left(\begin{array}{ccc}
\ell_{1} & \ell_{2} & \ell_{3} \\
m_{1} & m_{2} & m_{3}
\end{array}\right) \\
& \times\left[C_{\ell_{1} m_{1} \ell_{4} m_{3}}^{-1} C_{\ell_{2} m_{2} \ell_{5} m_{5}}^{-1} C_{\ell_{3} m_{3} \ell_{6} m_{6}}^{-1} a_{\ell_{4} m_{4}} a_{\ell_{5} m_{5}} a_{\ell_{6} m_{6}}\right. \\
& \left.-3 C_{\ell_{1} m_{1} \ell_{2} m_{2}}^{-1} C_{\ell_{3} m_{3} \ell_{4} m_{4}}^{-1} a_{\ell_{4} m_{4}}\right]
\end{aligned}
$$

where $F_{\mathcal{E}}$ is a constant which normalizes the estimator to have unit response to $B_{\ell_{1} \ell_{2} \ell_{3}}$. (The factor $1 / 6$ has been included for later convenience.) In order to translate the value of $\mathcal{E}$ to a constraint on the bispectrum amplitude, one needs to know both the normalization $F_{\mathcal{E}}$ and the variance $\operatorname{Var}(\mathcal{E})$.

The cubic term in the estimator can be thought of as a matched filter whose shape is given by the bispectrum $B_{\ell_{1} \ell_{2} \ell_{3}}$. The linear term can improve the variance of the estimator for certain bispectra (more precisely, bispectra of "squeezed" shape). For example, better limits on $f_{N L}^{\text {loc }}$ are obtained from the one-year WMAP data using an estimator containing the linear term than from the three-year WMAP data without the linear term [19, 47]. We note that for the fully optimal estimator (Eq. (54)), the data appears weighted by inverse signal + noise $C^{-1}$, and so the variance of the estimator always improves as more modes are added to the data. This is in contrast to suboptimal methods, such as those used to analyze WMAP data to date [19, 29, 44, 47], where as the cutoff multipole $\ell_{\max }$ is increased, the variance eventually worsens as a result of the inhomogeneities in the noise and the sky cuts.

We now introduce notation which will be used throughout the rest of the paper. Given a map $a=\left\{a_{\ell m}\right\}$, define

$$
T[a] \stackrel{\text { def }}{=} \frac{1}{6} \sum_{\ell_{i} m_{i}} B_{\ell_{1} \ell_{2} \ell_{3}}\left(\begin{array}{ccc}
\ell_{1} & \ell_{2} & \ell_{3} \\
m_{1} & m_{2} & m_{3}
\end{array}\right) a_{\ell_{1} m_{1}} a_{\ell_{2} m_{2}} a_{\ell_{3} m_{3}}
$$

We also consider the gradient of $T[a]$ with respect to the input map:

$$
\nabla_{\ell m} T[a] \stackrel{\text { def }}{=} \frac{\partial T[a]}{\partial a_{\ell m}^{*}}=\frac{1}{2} B_{\ell \ell_{2} \ell_{3}}\left(\begin{array}{ccc}
\ell & \ell_{2} & \ell_{3} \\
m & m_{2} & m_{3}
\end{array}\right) a_{\ell_{2} m_{2}}^{*} a_{\ell_{3} m_{3}}^{*}
$$


Note that $T[a]$ is a scalar which is cubic in the input map $a$, whereas $\nabla T[a]$ is another map which is quadratic in $a$.

The significance of $T$ is that three quantities of interest can be written compactly as Monte Carlo averages involving $T, \nabla T$. First, the estimator (Eq. (54)) can be rewritten:

$$
\begin{aligned}
\mathcal{E}[a]= & \frac{1}{F_{\mathcal{E}}}\left(T\left[C^{-1} a\right]-\right. \\
& \left.a_{\ell_{1} m_{1}} C_{\ell_{1} m_{1}, \ell_{2} m_{2}}^{-1}\left\langle\nabla_{\ell_{2} m_{2}} T\left[C^{-1} a^{\prime}\right]\right\rangle_{a^{\prime}}\right)
\end{aligned}
$$

obtaining the linear term as a Monte Carlo average. Here, $\langle\cdot\rangle_{a^{\prime}}$ denotes the Monte Carlo average taken over Gaussian realizations $a^{\prime}$ of signal + noise. Second, the normalization constant is given by:

$$
\begin{aligned}
F_{\mathcal{E}}= & \frac{1}{3}\left\langle\nabla_{\ell_{1} m_{1}} T\left[C^{-1} a\right] C_{\ell_{1} m_{1}, \ell_{2} m_{2}}^{-1} \nabla_{\ell_{2} m_{2}} T\left[C^{-1} a\right]\right\rangle_{a} \\
& -\frac{1}{3}\left\langle\nabla_{\ell_{1} m_{1}} T\left[C^{-1} a\right]\right\rangle_{a} C_{\ell_{1} m_{1}, \ell_{2} m_{2}}^{-1}\left\langle\nabla_{\ell_{2} m_{2}} T\left[C^{-1} a\right]\right\rangle_{a}
\end{aligned}
$$

Third, the estimator variance is given by

$$
\operatorname{Var}(\mathcal{E})=F_{\mathcal{E}}^{-1} .
$$

Eqs. (54)-(59) are derived in Appendix A. Taken together, they provide the basis for the following Monte Carlo procedure, which is the main result of this section:

1. In each Monte Carlo iteration, construct a random signal + noise realization $a$.

2. Evaluate $C^{-1} a, \nabla T\left[C^{-1} a\right]$, and $C^{-1}\left(\nabla T\left[C^{-1} a\right]\right)$ (see below).

3. Accumulate the contribution to the following Monte Carlo averages:

$$
\begin{gathered}
\left\langle\nabla_{\ell_{1} m_{1}} T\left[C^{-1} a\right]\right\rangle_{a} \\
\left\langle\nabla_{\ell_{1} m_{1}} T\left[C^{-1} a\right] C_{\ell_{1} m_{1}, \ell_{2} m_{2}}^{-1} \nabla_{\ell_{2} m_{2}} T\left[C^{-1} a\right]\right\rangle_{a}
\end{gathered}
$$

At the end of the Monte Carlo loop, the linear term in the estimator (Eq. (57)), the estimator normalization (Eq. (58)), and the estimator variance (Eq. (59)) have been computed. For each of these three quantities, the Monte Carlos converge to a value which incorporates the full noise covariance (including the sky cut), in contrast to methods which assume an $f_{\text {sky }}$ scaling. A feature of the method is that the variance (Eq. (59)) includes the effect of the linear term in the estimator, even though the linear term is not precomputed. Both are computed in parallel by the same set of Monte Carlos. After the Monte Carlo loop, one can evaluate the estimator (in the form given by Eq. (57D), including linear term and normalization, on the observed CMB map $m$.
In each Monte Carlo iteration, one $\nabla T$ evaluation and two multiplications by $C^{-1}$ are required. For the first of these ingredients, evaluating $\nabla T$, we will give a fast algorithm in the next section, assuming that the bispectrum satisfies the factorizability condition (Eq. (3D)).

A method for the second ingredient, multiplying a map by $(S+N)^{-1}$, will depend on the noise model of the experiment under consideration. In this paper, where our emphasis is on general algorithms which are not experiment-specific, we will not address this problem. However, let us emphasize that the experiment-specific challenge of finding an affordable method for $(S+N)^{-1}$ multiplication is a necessary ingredient in the optimal estimators of Eq. (50) and Eq. (54). If this problem can be solved, then the general framework we have presented here will permit optimal bispectrum estimation. Otherwise, one must fall back on a suboptimal estimator, e.g. replacing $C^{-1}$ in Eq. (54) by a filter which approximates it. Since it may not be feasible to solve the $(S+N)^{-1}$ problem for every dataset in which the three-point function is studied, we treat this case in App. B. including a discussion of how the linear term which improves the estimator variance may be retained, even when full optimality is lost.

We conclude this section by describing the generalization of our Monte Carlo procedure to joint estimation of multiple bispectra $B_{1}, \cdots, B_{n}$. Denote the quantity $T[a]$ (Eq. (55)), evaluated using bispectrum $B_{\alpha}$, by $T_{\alpha}[a]$. Then the optimal estimator for $B_{\alpha}$, debiased to have zero mean response to $B_{\beta}(\beta \neq \alpha)$, is given by:

$\mathcal{E}_{\alpha}=F_{\alpha \beta}^{-1}\left(T_{\beta}\left[C^{-1} a\right]-a_{\ell_{1} m_{1}} C_{\ell_{2} m_{2}}^{-1}\left\langle\nabla_{\ell_{2} m_{2}} T_{\beta}\left[C^{-1} a^{\prime}\right]\right\rangle_{a^{\prime}}\right)$

where $F_{\alpha \beta}$ is the matrix

$$
\begin{gathered}
F_{\alpha \beta}=\frac{1}{3}\left\langle\nabla_{\ell_{1} m_{1}} T_{\alpha}\left[C^{-1} a\right] C_{\ell_{1} m_{1}, \ell_{2} m_{2}}^{-1} \nabla_{\ell_{2} m_{2}} T_{\beta}\left[C^{-1} a\right)\left(\oint_{a} 3\right)\right. \\
-\frac{1}{3}\left\langle\nabla_{\ell_{1} m_{1}} T_{\alpha}\left[C^{-1} a\right]\right\rangle_{a} C_{\ell_{1} m_{1}, \ell_{2} m_{2}}^{-1}\left\langle\nabla_{\ell_{2} m_{2}} T_{\beta}\left[C^{-1} a\right]\right\rangle_{a}
\end{gathered}
$$

The estimator covariance is given by

$$
\operatorname{Cov}\left(\mathcal{E}_{\alpha}, \mathcal{E}_{\beta}\right)=F_{\alpha \beta}^{-1} \text {. }
$$

With multiple bispectra, the Monte Carlo procedure is the same as described above. In each iteration, $C^{-1} a$, $\nabla T_{\alpha}\left[C^{-1} a\right]$, and $C^{-1} \nabla T_{\alpha}\left[C^{-1} a\right]$ are computed, for a total of $n$ evaluations of $\nabla T$ and $(n+1)$ multiplications by $C^{-1}$.

The matrix $F_{\alpha \beta}$ defined in Eq. (64) is the $n$-by- $n$ Fisher matrix between the bispectra $B_{1}, \cdots, B_{n}$, with the full noise properties of the survey incorporated via the covariance $C$. (In the case where the noise is homogeneous, $F_{\alpha \beta}$ reduces to the Fisher matrix that was defined previously in Eq. (23)). In the estimator framework, $F_{\alpha \beta}$ arises as both the normalization (Eq. (62) ) and the covariance (Eq. (64)) of optimal bispectrum estimators. This is in complete analogy with optimal power spectrum estimation as described in $\$$ VIIA 


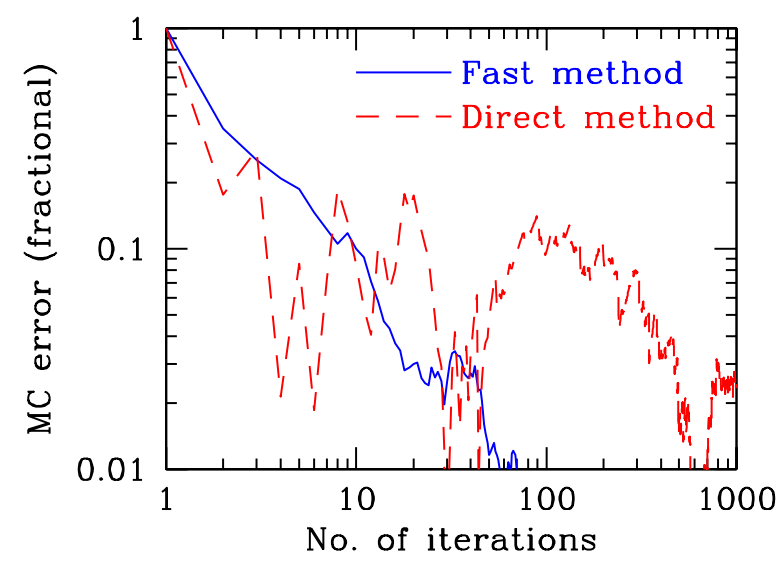

FIG. 6: Monte Carlo error after $N$ simulations, compared for one instance of the "fast" Monte Carlo method (Eq. (65)) and one instance of the "direct" method (Eq. (66) ), showing much faster convergence in the first case. The local form of the bispectrum (Eq. (13)) was used, with an all-sky homogeneous survey with $\ell_{\max }=500$, noise level $1000 \mu \mathrm{K}$-arcmin, and beam $\theta_{\mathrm{FWHM}}=10$ arcmin.

\section{Comparison with direct MC}

We have obtained the estimator covariance and normalization (which are equal for the case of the optimal estimator in Eq. (54)) as a Monte Carlo average (Eq. (58)). The reader may be wondering why a simpler Monte Carlo procedure was not used instead. For example, consider the variance of the cubic term $T\left[C^{-1} a\right]$ in the estimator. Following the treatment above, this would be computed via the Monte Carlo prescription

$$
\begin{aligned}
\operatorname{Var} & \left.T\left[C^{-1} a\right]\right)= \\
& \frac{1}{3}\left\langle\nabla_{\ell_{1} m_{1}} T\left[C^{-1} a\right] C_{\ell_{1} m_{1}, \ell_{2} m_{2}}^{-1} \nabla_{\ell_{2} m_{2}} T\left[C^{-1} a\right]\right\rangle_{a} \\
& +\frac{2}{3}\left\langle\nabla_{\ell_{1} m_{1}} T\left[C^{-1} a\right]\right\rangle_{a} C_{\ell_{1} m_{1}, \ell_{2} m_{2}}^{-1}\left\langle\nabla_{\ell_{2} m_{2}} T\left[C^{-1} a\right]\right\rangle_{a}
\end{aligned}
$$

(Compare Eq. (58), which gives the variance when the linear term is included in addition to the cubic term.) Why not compute this more simply by using the "direct" Monte Carlo prescription

$$
\operatorname{Var}\left(T\left[C^{-1} a\right]\right)=\left\langle T\left[C^{-1} a\right] T\left[C^{-1} a\right]\right\rangle_{a}
$$

instead?

We have found that the convergence of the first expression (Eq. (65) ) is much more rapid than the second (Eq. (66) ). This is illustrated in Fig. 6, where we show the dependence of the error on the number of Monte Carlo iterations, for both prescriptions. It is seen that the Monte Carlo framework we have given above converges much more quickly than "direct" Monte Carlo, requiring only $\sim 100$ simulations to reach $1 \%$ accuracy. For this reason, the Monte Carlo framework presented above is preferable to direct Monte Carlo computation of the estimator variance, even though the computational cost per iteration is doubled (since $\nabla T$ must be computed instead of $T$, and two $C^{-1}$ multiplications are needed instead of one). Another benefit is that the linear term in the optimal estimator is computed in parallel.

It may seem suprising that the convergence rate of the two Monte Carlo prescriptions is so different. One way to see this intuitively is to note that the first expression (Eq. 65) contains two fewer powers of the map $a$ than the second expression (Eq. ([66) $)$. In effect, one factor of $\left(a a^{T}\right)$ has been replaced "in advance" by its Monte Carlo average $C$, thus accelerating convergence. (Note that the same phenomenon exists in the context of power spectrum estimation; if the Fisher matrix is computed by Monte Carlo trace evaluation as in Eq. (52), the convergence rate will be much faster than estimating the covariance of the estimator in Eq. (50) by direct Monte Carlo.)

\section{EVALUATION OF $T, \nabla T$}

We have now described a general Monte Carlo framework for optimal bispectrum estimation in the presence of sky cuts and inhomogeneous noise, with two ingredients deferred: a method for evaluating $\{T, \nabla T\}$, and a method for multipying a map by $(S+N)^{-1}$. In this section, we address the first of these. We present a fast algorithm which, given an input set of multipoles $a=\left\{a_{\ell m}\right\}$ and bispectrum written in factorizable form

$$
b_{\ell_{1} \ell_{2} \ell_{3}}=\frac{1}{6} \sum_{i=1}^{N_{\text {fact }}} X_{\ell_{1}}^{(i)} Y_{\ell_{2}}^{(i)} Z_{\ell_{3}}^{(i)}+\text { (symm.) }
$$

evaluates $T[a]$ and $\nabla T[a]$.

If $X_{\ell}$ is any $\ell$-dependent quantity, define $X_{a}(\mathbf{x})$ to be the position-space map obtained by applying the filter $X_{\ell}$ to $a_{\ell m}$ :

$$
X_{a}(\mathbf{x})=\sum_{\ell m} X_{\ell} a_{\ell m} Y_{\ell m}(\mathbf{x})
$$

The basis for our algorithm will be the following positionspace expression for $T[a]$ :

$T[a]=\frac{1}{6} \sum_{i=1}^{N_{\text {fact }}} \int d \cos (\theta) d \varphi X_{a}^{(i)}(\theta, \varphi) Y_{a}^{(i)}(\theta, \varphi) Z_{a}^{(i)}(\theta, \varphi)$

obtained from Eq. (67) and the identity

$$
\begin{gathered}
\int d \cos (\theta) d \varphi Y_{\ell_{1} m_{1}}(\theta, \varphi) Y_{\ell_{2} m_{2}}(\theta, \varphi) Y_{\ell_{3} m_{3}}(\theta, \varphi) \\
=\left[\frac{\left(2 \ell_{1}+1\right)\left(2 \ell_{2}+1\right)\left(2 \ell_{3}+1\right)}{4 \pi}\right]^{1 / 2} \\
\times\left(\begin{array}{ccc}
\ell_{1} & \ell_{2} & \ell_{3} \\
m_{1} & m_{2} & m_{3}
\end{array}\right)\left(\begin{array}{ccc}
\ell_{1} & \ell_{2} & \ell_{3} \\
0 & 0 & 0
\end{array}\right)
\end{gathered}
$$


We next observe that the integral in Eq. (69) can be done exactly using Gauss-Legendre quadrature in $\cos (\theta)$ with $N_{\theta}=\left\lfloor 3 \ell_{\max } / 2\right\rfloor+1$ points, and uniform quadrature in $\varphi$ with $N_{\varphi}=\left(3 \ell_{\max }+1\right)$ points. (This observation is the basis for the GLESP pixelization [48].) This is because each term in the integrand is a polynomial in $\cos (\theta)$ of degree $\leq 3 \ell_{\max }$, multiplied by a factor $e^{i m \varphi}$ with $-3 \ell_{\max } \leq m \leq 3 \ell_{\max }$. (There is a subtlety here: some terms in Eq. (69) contain odd powers of $\sqrt{1-z^{2}}$, for which Gauss-Legendre integration is not exact, but each such term has an odd value of $m$, and hence gives zero when integrated over $\varphi$.)

This observation permits the integral to be replaced by a finite sum without approximation:

$$
T[a]=\frac{\pi}{3 N_{\varphi}} \sum_{i=1}^{N_{\text {fact }}} \sum_{\theta, \varphi} w_{\theta} X_{a}^{(i)}(\theta, \varphi) Y_{a}^{(i)}(\theta, \varphi) Z_{a}^{(i)}(\theta, \varphi)
$$

where $w_{\theta}$ denotes the Gauss-Legendre weight at the quadrature point $\theta$.

Our algorithm for evaluating $T[a]$, in the form (71), is given as follows. There is an outer loop over $\theta$ in which the contribution of each isolatitude ring to the integral is accumulated. Within this loop (i.e. for fixed $\theta$ ), the first step is to fill the matrix

$$
\mathbf{M}_{\ell m}=a_{\ell m} Y_{\ell m}(\theta, 0)
$$

using upward recursion in $\ell$ to generate the spherical harmonics. Second, we evaluate the matrix

$$
\mathbf{N}_{\ell \varphi}=\sum_{m} \mathbf{M}_{\ell m} e^{i m \varphi}
$$

by taking an FFT along each column of $\mathbf{M}$. Third, we evaluate

$$
\mathbf{X}_{i \varphi}=\sum_{\ell} X_{\ell}^{(i)} \mathbf{N}_{\ell \varphi}
$$

by matrix multiplication. After this step, the matrix entry $\mathbf{X}_{i \varphi}$ is equal to the quantity $X_{a}^{(i)}(\theta, \varphi)$ defined in Eq. (68). We compute matrices $\mathbf{Y}_{i \varphi}, \mathbf{Z}_{i \varphi}$ analagously, replacing $X_{\ell}^{(i)}$ in Eq. (74) by $Y_{\ell}^{(i)}, Z_{\ell}^{(i)}$. The fourth step is to accumulate the contribution of one isolatitude ring (in Eq. (710) to $T[a]$ as follows:

$$
T[a] \leftarrow T[a]+\frac{\pi w_{\theta}}{3 N_{\varphi}} \sum_{i, \varphi} \mathbf{X}_{i \varphi} \mathbf{Y}_{i \varphi} \mathbf{Z}_{i \varphi}
$$

This completes the algorithm for evaluating $T$; we now describe extra steps needed to evaluate $\nabla T$. The idea is to compute derivatives of $T$ with respect to each of the quantities defined in Eqs. (72)-(75), in reverse order. First, differentiating Eq. (75), one computes

$$
\frac{\partial T}{\partial \mathbf{X}_{i \varphi}}=\frac{\pi w_{\theta}}{3 N_{\varphi}} \mathbf{Y}_{i \varphi} \mathbf{Z}_{i \varphi}
$$

and likewise for $\left(\partial T / \partial \mathbf{Y}_{i \varphi}\right)$ and $\left(\partial T / \partial \mathbf{Z}_{i \varphi}\right)$. Similarly, by differentiating Eqs. (72)-(174), one computes the following quantities in order:

$$
\begin{gathered}
\frac{\partial T}{\partial \mathbf{N}_{\ell \varphi}}=\sum_{i} X_{\ell}^{(i)} \frac{\partial T}{\partial \mathbf{X}_{i \varphi}}+Y_{\ell}^{(i)} \frac{\partial T}{\partial \mathbf{Y}_{i \varphi}}+Z_{\ell}^{(i)} \frac{\partial T}{\partial \mathbf{Z}_{i \varphi}} \\
\frac{\partial T}{\partial \mathbf{M}_{\ell m}}=\sum_{\varphi} \frac{\partial T}{\partial \mathbf{N}_{\ell \varphi}} e^{i m \varphi} \\
\frac{\partial T}{\partial a_{\ell m}}=\frac{\partial T}{\partial \mathbf{M}_{\ell m}} Y_{\ell m}(\theta, 0)
\end{gathered}
$$

The final result gives the contribution of one isolatitude ring to $\nabla_{\ell m} T=\left(\partial T / \partial a_{\ell m}^{*}\right)$. This procedure computes $\{T[a], \nabla T[a]\}$ in twice the running time needed to compute $T[a]$ alone.

The algorithm we have just presented was closely inspired by the position-space estimator of Komatsu, Spergel and Wandelt [18], in the context of the local bispectrum (Eq. (13)). Indeed, our algorithm can be viewed as both generalizing the KSW estimator to an arbitrary input bispectrum satisfying the factorizability condition, and permitting calculation of the gradient $\nabla T[a]$ in addition to $T[a]$. (We have seen that Monte Carlo evaluations of $\nabla T$ are needed to compute the linear term in the estimator, the normalization $F_{\mathcal{E}}$, and the variance $\operatorname{Var}(\mathcal{E})$ in the presence of sky cuts and inhomogeneous noise.)

Additionally, we have presented the details of the algorithm in an optimized way which dramatically improves the running time, compared to existing implementations. For example, in [19], a running time of 60 CPU-minutes is quoted to evaluate the cubic term $T[a]$ for the local bispectrum (Eq. (13)), using a quadrature in the $r$ integral with 260 points, at $\ell_{\max }=335$. With these parameters, our implementation evalues $T[a]$ in $27 \mathrm{CPU}$-seconds; after optimzing $N_{\text {fact }}$ using the method of $₫ \mathbb{V}$, this is further improved to $4 \mathrm{CPU}$-seconds.

The main reason that our implementation is so fast is that the only steps with $\operatorname{cost} \mathcal{O}\left(N_{\text {fact }} \ell_{\text {max }}^{3}\right)$ have been written as matrix multiplications in Eqs. (74), (77). These can be evaluated extremely efficiently using an optimzed library such as BLAS. In existing implementations, the same asymptotic cost is accrued by means of $N_{\text {fact }}$ separate $\mathcal{O}\left(\ell_{\max }^{3}\right)$ spherical harmonic transforms, but the overall prefactor is much larger. A second, less important, optimization is that we have converted the integral (Eq. (69)) to a sum (Eq. (71)) using GaussLegendre quadrature in $\cos (\theta)$ and uniform quadrature in $\varphi$, rather than using a pixelization such as Healpix. In addition to giving an exact evaluation of Eq. (55), our "pixelization" is optimized to minimize the number of quadrature points needed, which translates to smaller matrix sizes in the rate-limiting steps.

In Tab. $\mathrm{Q}$, we have shown timings for one $T[a]$ evaluation in several mock surveys. (Note that in the Monte Carlo framework for \$VII several such evaluations are needed in each Monte Carlo iteration.) Using the optimizations that we have presented here, which improve 


\begin{tabular}{|c||c|c|}
\hline & WMAP3 & Planck \\
\hline & $\left(\ell_{\max }=1000\right)$ & $\left(\ell_{\max }=2000\right)$ \\
\hline Point source (Eq. (7)) & 3 CPU-sec & 1 CPU-min \\
ISW-lensing (Eq. (4) $)$ & 10 CPU-sec & 2 CPU-min \\
Local (Eq. (12)) & 84 CPU-sec & 14 CPU-min \\
Equilateral (Eq. (22) & 117 CPU-sec & 23 CPU-min \\
Gravitational (Eq. (16) $)$ & 467 CPU-sec & 81 CPU-min \\
HD (Eq. (19) $)$ & 149 CPU-sec & 38 CPU-min \\
\hline
\end{tabular}

TABLE V: CPU time needed for one evaluation of $T[a]$, for each of the optimized bispectra from Tab. III Evaluating $\nabla T[a]$ in addition to $T[a]$ would double the running times shown.

existing implementations by several orders of magnitude, a fully optimal bispectrum analysis for Planck $\left(\ell_{\max }=\right.$ 2000) should easily be feasible.

\section{SIMULATING NON-GAUSSIAN MAPS}

So far, our emphasis has been on bispectrum estimation; however, another application of our machinery is that it provides a fast algorithm for simulating nonGaussian maps. It should be emphasized from the outset that there is no "universal" probability distribution for a field whose power spectrum and bispectrum are prescribed. This is because the four- and higher-point connected correlation functions must be nonvanishing, for the probability density to be positive definite. In general, two schemes for simulating a non-Gaussian field, with the same power spectrum and bispectrum, will differ in their higher-point amplitudes. However, we expect that our algorithm will be useful in the regime of weak non-Gaussianity, where higher-point amplitudes can be neglected.

We present a simulation algorithm which generates allsky simulated maps starting from arbitrary input power spectrum $C_{\ell}$, and any input bispectrum $B_{\ell_{1} \ell_{2} \ell_{3}}$ which satisfies the factorizability condition (Eq. (3)). The power spectrum and bispectrum of the field $a_{\ell m}^{\prime}$ which is simulated will satisfy:

$$
\begin{aligned}
C_{\ell}^{\prime} & =C_{\ell}+\mathcal{O}\left(B^{2}\right) \\
B_{\ell_{1} \ell_{2} \ell_{3}}^{\prime} & =B_{\ell_{1} \ell_{2} \ell_{3}}+\mathcal{O}\left(B^{3}\right)
\end{aligned}
$$

where $\mathcal{O}\left(B^{k}\right)$ denotes terms containing $k$ or more powers of the bispectrum. For $N \geq 4$, the connected $N$-point function of the simulated field will satisfy:

$$
\left\langle a_{\ell_{1} m_{1}} a_{\ell_{2} m_{2}} \cdots a_{\ell_{N} m_{N}}\right\rangle_{\text {conn. }}=\mathcal{O}\left(B^{N-2}\right)
$$

Under the assumption of weak non-Gaussianity, where the extra terms in Eq. (81) can be neglected, the power spectrum and bispectrum of the simulated field will agree with the input values $C_{\ell}, B_{\ell_{1} \ell_{2} \ell_{3}}$. The problem of simulating non-Gaussian fields has received some attention in the literature [29 32], but no method has been proposed with this generality.

Our simulation algorithm is given as follows. One first simulates a Gaussian random field $a_{\ell m}$ with power spectrum $C_{\ell}$. Then define $a_{\ell m}^{\prime}$ by perturbing to order $\mathcal{O}(B)$ as follows:

$$
a_{\ell m}^{\prime}=a_{\ell m}+\frac{1}{3} \nabla_{\ell m} T\left[C_{\ell^{\prime}}^{-1} a_{\ell^{\prime} m^{\prime}}\right]
$$

The algorithm given in $\$$ VIII is used to evaluate $\nabla T$. The CPU time needed for one random realization of $a_{\ell m}^{\prime}$ is therefore given by Tab. $\mathrm{V}$ (with a factor of two included for calculating $\nabla T$ in addition to $T$ ), e.g. $168 \mathrm{CPU}$ seconds for the local bispectrum at WMAP3 noise levels and $\ell_{\max }=1000$. With this level of performance, nonGaussian simulations can easily be included in a Monte Carlo analysis of Planck data, in the generality of an arbitrary factorizable bispectrum.

To lowest (zeroth) order in $B$, the power spectrum of $a_{\ell m}^{\prime}$ is $C_{\ell}$. Let us calculate the lowest-order contribution to the bispectrum of $a_{\ell m}^{\prime}$. Plugging in the defintion (Eq. (56) ) of $\nabla T$,

$$
\begin{aligned}
& \left\langle a_{\ell_{1} m_{1}}^{\prime} a_{\ell_{2} m_{2}}^{\prime} a_{\ell_{3} m_{3}}^{\prime}\right\rangle \\
& =\frac{1}{6} B_{\ell_{1} \ell_{2}^{\prime} \ell_{3}^{\prime}}\left(\begin{array}{ccc}
\ell_{1} & \ell_{2}^{\prime} & \ell_{3}^{\prime} \\
m_{1} & m_{2}^{\prime} & m_{3}^{\prime}
\end{array}\right) \times \\
& \quad C_{\ell_{2}^{\prime}}^{-1} a_{a_{2}^{\prime} m_{2}^{\prime}} C_{\ell_{3}^{\prime}}^{-1} a_{\ell_{3}^{\prime} m_{3}^{\prime}}^{*} a_{\ell_{2} m_{2}} a_{\ell_{3} m_{3}}+\text { (symm.) } \\
& =B_{\ell_{1} \ell_{2} \ell_{3}}\left(\begin{array}{ccc}
\ell_{1} & \ell_{2} & \ell_{3} \\
m_{1} & m_{2} & m_{3}
\end{array}\right)
\end{aligned}
$$

where + (symm.) denotes the sum over five additional terms obtained by permuting $\left(\ell_{i}, m_{i}\right)$. This shows that the lowest-order bispectrum is simply $B_{\ell_{1} \ell_{2} \ell_{3}}$; it is easy to see that the orders of the higher-order terms are as claimed in Eq. 81).

In Fig. 7. we illustrate the method by showing a single random realization, split into three pieces. From Eq. (83), each realization is generated as a Gaussian piece (the first term on the right-hand side) plus a small nonGaussian perturbation (the second term) which depends on the bispectrum. We have shown the Gaussian piece separately in Fig. 7, and also shown the non-Gaussian term for the case of the local (Eq. (12) ) and equilateral (Eq. (22)) bispectra.

Let us emphasize the caveats associated with the simulation algorithm presented here. A model which predicts non-Gaussianity at the three-point level will also predict higher-point connected correlation functions; these will not be reproduced faithfully by the simulations. If higher-point correlations are important, then one must tailor the simulation method to the specific model; one cannot expect to use a "generic" algorithm which only incorporates two-point and three-point information, such as the algorithm we have given in this section. However, in the regime of weak non-Gaussianity, where the three- 


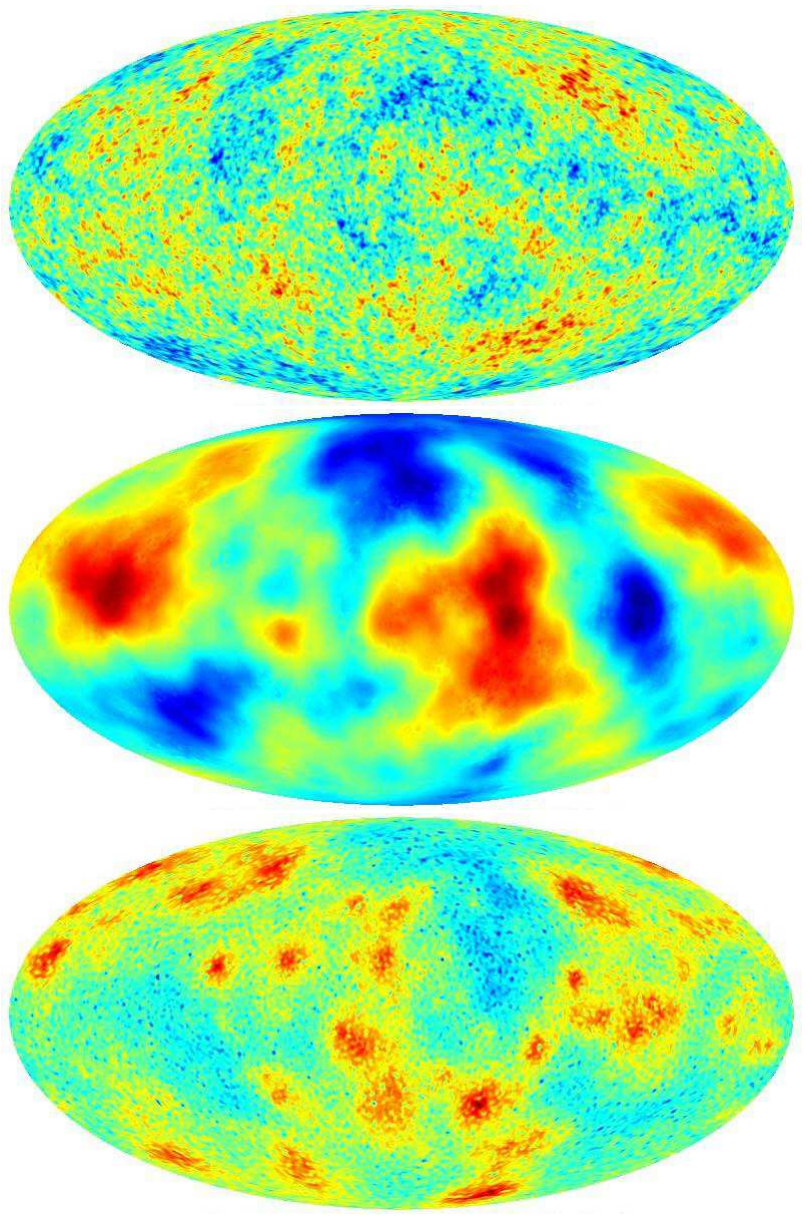

FIG. 7: One random realization obtained using the simulation algorithm from $₫ \mathrm{IX}$. consisting of a Gaussian map $\left\{a_{\ell m}\right\}$ (top), and non-Gaussian maps $\left\{a_{\ell m}^{\text {loc }}\right\}$ (middle), $\left\{a_{\ell m}^{\text {eq }}\right\}$ (bottom). To lowest order, the map $\left(a_{\ell m}+f_{N L}^{\text {loc }} a_{\ell m}^{\text {loc }}+f_{N L}^{\text {eq }} a_{\ell m}^{\text {eq }}\right)$ will have the power spectrum of the fiducial model, and bispectrum which is a linear combination of the local and equilateral forms (Eqs. (12), (22)). All three maps have been smoothed with a $1^{\circ}$ Gaussian beam.

point function is marginal and higher-point correlations are negligible, our simulation method should apply.

For the specific case of the local bispectrum $B_{\ell_{1} \ell_{2} \ell_{3}}^{\text {loc }}$, the generic simulation algorithm has another caveat: the non-Gaussian contribution to the power spectrum, while formally of order $\mathcal{O}\left(f_{N L}^{2}\right)$, os unphysically large even for moderate values of $f_{N L}$. This is interpreted physically and discussed in more detail in the appendix of [49], where a modification of the generic algorithm is proposed for the local shape, to eliminate the spuriously large non-Gaussian power spectrum. (For the case of the local shape, there is also an exact simulation algorithm which correctly simulates all higher-point statistics, at the expense of somewhat increased computational cost [30].)

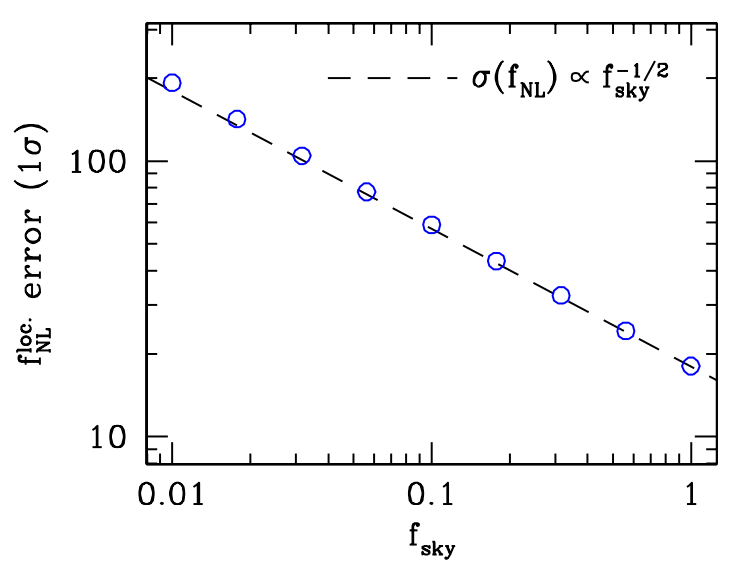

FIG. 8: Dependence of the $1 \sigma$ error $\sigma\left(f_{N L}^{\text {loc }}\right)$ on $f_{\text {sky }}$, for a spherical cap shaped survey with noise level $500 \mu \mathrm{K}$-arcmin and 10 arcmin beam, showing good agreement between the Monte Carlo errors (circles), and simple $f_{\text {sky }}^{-1 / 2}$ scaling (dotted line).

\section{EXAMPLE SURVEYS}

The general Monte Carlo framework for optimal bispectrum estimation which has been presented above applies to any survey for which a map can be efficiently multiplied by $C^{-1}$. We conclude by considering some mock surveys whose sky coverage and noise are azimuthally symmetric. The role of azimuthal symmetry is to make the noise covariance matrix diagonal in $m$, so that $C^{-1}$ multiplication can be performed quickly by "brute force" 22].

Our first example will be a survey with homogeneous noise level $500 \mu \mathrm{K}$-arcmin and Gaussian beam $\theta_{\mathrm{FWHM}}=$ 10 arcmin, with the geometry of a spherical cap. In Fig. 8 we show $1 \sigma$ errors $\sigma\left(f_{N L}^{\text {loc }}\right)$ obtained using the optimal estimation framework from $\oint \mathrm{VII}$, for varying $f_{\text {sky }}$. It is seen that, with optimal estimators and for this simple sky cut, $\sigma\left(f_{N L}^{\text {loc }}\right)$ varies as $f_{\text {sky }}^{-1 / 2}$ over two orders of magnitude.

As a second example, we consider a Planck-like survey. To approximate Planck within the constraint of azimuthal symmetry, we include a "galactic" sky cut which masks an equatorial band whose size is chosen to give $f_{\text {sky }}=0.8$, and take a pixel-dependent noise variance of the form

$$
\sigma(\theta, \varphi)=\left(\frac{2}{\pi}\right) \sigma_{0}^{2} \sin (\theta)
$$

where the average noise level $\sigma_{0}$ is determined by the sensitivities in Tab. I] (This angular dependence of the noise is motivated by the Planck scan strategy, which scans along great circles through the ecliptic poles; however, note that azimuthal symmetry requires us to place the poles perpendicular to the galactic cut.)

Since it is currently unclear what cutoff in $\ell$ will be 


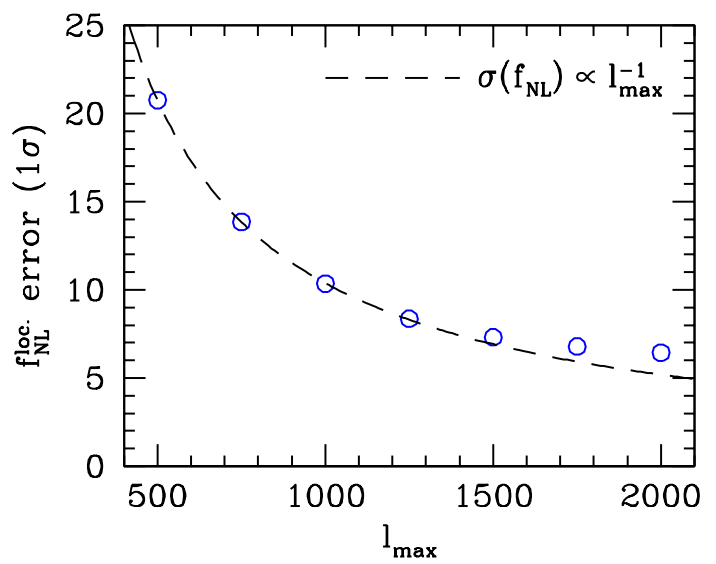

FIG. 9: Dependence of the $1 \sigma$ error $\sigma\left(f_{N L}^{\text {loc }}\right)$ on $\ell_{\max }$, for the azimuthally symmetric approximation to Planck described in $\$ \mathrm{X}$, showing an $\ell_{\max }^{-1}$ dependence throughout the sample variance limited regime.

needed in Planck to ensure that foreground contamination is sufficiently well-controlled for bispectrum estimation, we show the dependence of $\sigma\left(f_{N L}^{\text {loc }}\right)$ on $\ell_{\max }$ in Fig. 9. It is seen that, throughout the range of multipoles where Planck is sample variance limited $(\ell \lesssim 1500), \sigma\left(f_{N L}^{\text {loc }}\right)$ varies roughly as $\ell_{\max }^{-1}$, as expected from [34]. At higher $\ell, \sigma\left(f_{N L}^{\text {loc }}\right)$ flattens as instrumental noise becomes a contaminant. At $\ell_{\max }=2000$, the largest value considered, the $1 \sigma$ error agrees with the Fisher matrix forecast from @VI. This demonstrates the ability of the optimal estimator to saturate statistical limits on $\sigma\left(f_{N L}^{\text {loc }}\right)$ in the presence of sky cuts and inhomogeneous noise. Another feature seen in Fig. 9 is that $\sigma\left(f_{N L}^{\text {loc }}\right)$ always decreases as $\ell_{\max }$ increases. This is a characteristic of optimal estimators, which can never worsen as more modes are added. In contrast, for non-optimal bispectrum estimators, the variance eventually begins to worsen when $\ell_{\max }$ becomes large enough that not all modes are signal-dominated [11, 47].

\section{DISCUSSION}

Perhaps the most fundamental problem in connecting a predicted shape of the CMB bispectrum with data is that analysis techniques which allow an arbitrary angular bispectrum $B_{\ell_{1} \ell_{2} \ell_{3}}$ are computationally prohibitive. For example, even with the simplifying assumption of all-sky homogeneous noise, the cost of evaluating an optimal estimator is $\mathcal{O}\left(\ell_{\max }^{5}\right)$, due to the number of nonzero terms in the harmonic-space sum (Eq. (54)). In practice, this problem has meant that bispectrum estimation to date has been limited to a few special forms of the bispectrum, such as the "local" shape (Eq. (12)), where fast estimators are available. On the other hand, there is a growing menagerie of theoretically motivated bispectra from secondary anisotropies [14 17, 37, 38] and early universe physics [6 10], which one might wish to study in a dataset such as Planck.

We have shown that the factorizability criterion for bispectra (Eq. (3)) is a compromise in generality which is specific enough to enable fast algorithms, yet general enough to encompass a wide range (\$III) of predicted shapes for $B_{\ell_{1} \ell_{2} \ell_{3}}$. We have given several fast algorithms which operate in the generality of an arbitrary factorizable bispectrum. In each case, the idea behind the algorithm is that the factorizability condition permits efficient computation by translating from harmonic to position space.

The first such algorithm is an optimization algorithm which, given an input bispectrum written as a sum of many factorizable terms, outputs an "optimized" bispectrum with fewer terms which closely approximates the input. It is feasible to run this algorithm on very large input sizes; e.g. the higher-derivative bispectrum for Planck (with $N_{\text {fact }}=80000$ and $\ell_{\max }=2000$ ) was treated, using a two-level optimization procedure described in $8 \mathrm{~V}$. In this example, the optimization algorithm outputs a bispectrum which has only 86 factorizable terms, and is provably indistinguishable from the input; the algorithm is only allowed to terminate when the Fisher distance between the two is $\leq 10^{-6}$.

Second, we have given a general Monte Carlo based framework for optimal bispectrum estimation, which relies on two ingredients: a method for computing the quantities $\left\{T[a], \nabla_{\ell m} T[a]\right\}$ defined in Eq. (55), and a method for multiplying a map $a=\left\{a_{\ell m}\right\}$ by $(S+N)^{-1}$, where $S$ and $N$ are signal and noise covariance matrices. For the first of these, we have given a fast algorithm which can be thought of as generalizing the KSW estimator (constructed in [18] for the local shape) to any factorizable bispectrum, and providing a convenient way to compute the linear term in the optimal estimator (Eq. (54)). Additionally, we have described optimizations, such as rewriting the slowest steps as matrix multiplications, which improve the running time of existing implementations by several orders of magnitude. This speedup allows us to use large values of $\ell_{\max }$ and should make a Monte Carlo analysis for Planck very affordable (Tab. V).

Our estimator is fully optimal under the assumption that an affordable method can be found for multiplying a CMB map by $(S+N)^{-1}$, where $S$ and $N$ are signal and noise covariances respectively. Finding such a method will depend on the noise model and is outside the scope of this paper, where our emphasis has on algorithmic aspects of bispectrum estimation and simulation which are not experiment-specific. If no such method can be found, then our estimator is not fully optimal, but still includes the linear term, which should improve constraints in the presence of inhomogeneous noise (App. B). The " $C^{-1}$ problem" is a general ingredient in optimal estimators and also arises, e.g. for optimal power spectrum estimation and for lens reconstruction. We will discuss this problem, with emphasis on features of the noise model 
which arise in WMAP, in a forthcoming paper analyzing three-year WMAP data.

Finally, we have given a simulation algorithm which generates random sky maps with prescribed power spectrum and factorizable bispectrum. This greatly extends the generality of existing methods for simulating nonGaussian fields, and is computationally inexpensive, easily permitting non-Gaussian simulations to be included in Monte Carlo based pipelines if needed. An important caveat is that the higher-point correlation functions are not guaranteed to match the model which gives rise to the bispectrum; the higher correlations are merely guaranteed to be small (Eq. (82)). Therefore, the simulation method should only be relied upon in the regime of weak non-Gaussianity, where higher correlations are negligible.

As an application of these techniques, we have done a Fisher matrix forecast for multiple bispectra at Planck sensitivities ( $\sqrt{\mathrm{VI}}$ ). Of the bispectra considered, we found that four were nondegenerate: the point source, ISWlensing, local, and equilateral shapes. Correlations between these four are small, so that the shapes can be independently constrained. However, at Planck sensitivity levels, the ISW-lensing bispectrum can still significantly bias estimates of the local bispectrum, if not marginalized in the analysis. We have also demonstrated the optimal estimator on example surveys $(\widehat{X})$, showing that it is both computationally affordable and achieves statistical limits on $\sigma\left(f_{N L}^{\text {loc }}\right)$ for a Planck-like survey with inhomogeneous noise and $\ell_{\max }=2000$.

Our most general conclusion is that the factorizability criterion (Eq. (3) ) is a promising approach for bridging the gap between a theoretically motivated shape of the bispectrum and data. We have described a generic "toolkit", with algorithms for Fisher forecasting, analysis, and simulation, which can be implemented once and subsequently applied to any bispectrum which can be written in factorizable form. Even if the number of factorizable terms appears to be intractably large $\left(\sim 10^{5}\right)$, the optimization algorithm ( $(\nabla)$ can still be used and may reduce the number of terms to a manageable level, as in the case of the higher-derivative shape.

\section{Acknowledgments}

We would like to thank Wayne Hu, Dragan Huterer, Michele Liguori, Eugene Lim and Bruce Winstein for useful discussions. We acknowledge use of the FFTW, LAPACK, CAMB, and Healpix software packages. KMS was supported by the Kavli Institure for Cosmological Physics through the grant NSF PHY-0114422. MZ was supported by the Packard and Sloan foundations, NSF AST-0506556 and NASA NNG05GG84G.

\section{Appendix A: Monte Carlo averages}

The purpose of this appendix is to derive Eqs. (57), (58), and (59) in 8VII, in which Monte Carlo expressions are given for the linear term in the estimator $\mathcal{E}$, the normalization $F_{\mathcal{E}}$, and the variance $\operatorname{Var}(\mathcal{E})$. We note that Monte Carlo averages involving $\left(C^{-1} a\right)$, where $a$ is a Gaussian random field with covariance $C$, can be evaluated using the contraction:

$$
\left(C^{-1} a\right)_{\ell_{1} m_{1}}\left(C^{-1} a\right)_{\ell_{2} m_{2}}=C_{\ell_{1} m_{1}, \ell_{2} m_{2}}^{-1} .
$$

It will be convenient to define the following quantities:

$$
\begin{aligned}
\alpha \stackrel{\text { def }}{=} & B_{\ell_{1} \ell_{2} \ell_{3}} B_{\ell_{4} \ell_{5} \ell_{6}}\left(\begin{array}{ccc}
\ell_{1} & \ell_{2} & \ell_{3} \\
m_{1} & m_{2} & m_{3}
\end{array}\right)\left(\begin{array}{ccc}
\ell_{4} & \ell_{5} & \ell_{6} \\
m_{4} & m_{5} & m_{6}
\end{array}\right) \\
& \times\left(C^{-1}\right) \ell_{\ell_{1} m_{1}, \ell_{4} m_{4}}\left(C^{-1}\right) \ell_{2} m_{2}, \ell_{5} m_{5} \\
\beta_{\ell m} \stackrel{\text { def }}{=} & B_{\ell \ell_{2} \ell_{3}}\left(\begin{array}{ccc}
\ell & \ell_{2} & \ell_{3} \\
m & m_{2} & m_{3} m_{3}, \ell_{6} m_{6}
\end{array}\right) C_{\ell_{2} m_{2}, \ell_{3} m_{3}}^{-1}
\end{aligned}
$$

In terms of these, we evaluate the following Monte Carlo averages, using the definition (Eq. (55)) of $T$ :

$$
\begin{gathered}
\left\langle\nabla_{\ell m} T\left[C^{-1} a\right]\right\rangle_{a}=\frac{1}{2} B_{\ell \ell_{2} \ell_{3}}\left(\begin{array}{ccc}
\ell & \ell_{2} & \ell_{3} \\
m & m_{2} & m_{3}
\end{array}\right) \\
\times\left\langle\left(C^{-1} a\right)_{\ell_{2} m_{2}}\left(C^{-1} a\right)_{\ell_{3} m_{3}}\right\rangle_{a} \\
=\frac{1}{2} \beta_{\ell m} . \\
\left\langle\nabla_{\ell_{1} m_{1}} T\left[C^{-1} a\right] C_{\ell_{1} m_{1}, \ell_{2} m_{2}}^{-1} \nabla_{\ell_{2} m_{2}} T\left[C^{-1} a\right]\right\rangle_{a} \\
=\frac{1}{4} B_{\ell_{1} \ell_{3} \ell_{4}} B_{\ell_{2} \ell_{5} \ell_{6}}\left(\begin{array}{ccc}
\ell_{1} & \ell_{3} & \ell_{4} \\
m_{1} & m_{3} & m_{4}
\end{array}\right)\left(\begin{array}{ccc}
\ell_{2} & \ell_{5} & \ell_{6} \\
m_{2} & m_{5} & m_{6}
\end{array}\right) \\
\times C_{\ell_{1} m_{1}, \ell_{2} m_{2}}^{-1}\left\langle\left(C^{-1} a\right)_{\ell_{3} m_{3}}\left(C^{-1} a\right)_{\ell_{4} m_{4}}\right. \\
\left(\begin{array}{cc}
\left.\left(C^{-1} a\right)_{\ell_{5} m_{5}}\left(C^{-1} a\right)_{\ell_{6} m_{6}}\right\rangle_{a} \\
=\frac{\alpha}{2}+\frac{1}{4} \beta_{\ell_{1} m_{1}} C_{\ell_{1} m_{1}, \ell_{2} m_{2}}^{-1} \beta_{\ell_{2} m_{2} .} .
\end{array}\right.
\end{gathered}
$$

The Monte Carlo expression (Eq. (57)) for $\mathcal{E}$ follows immediately from the definition (Eq. (54) ) and Eq. (A3) above.

Turning next to the estimator normalization $F_{\mathcal{E}}$, the definition (Eq. (54)) implies:

$$
\begin{aligned}
F_{\mathcal{E}}= & \frac{1}{6} B_{\ell_{1} \ell_{2} \ell_{3}} B_{\ell_{4} \ell_{5} \ell_{6}}\left(\begin{array}{ccc}
\ell_{1} & \ell_{2} & \ell_{3} \\
m_{1} & m_{2} & m_{3}
\end{array}\right)\left(\begin{array}{ccc}
\ell_{4} & \ell_{5} & \ell_{6} \\
m_{4} & m_{5} & m_{6}
\end{array}\right) \\
& \times\left(C^{-1}\right) \ell_{\ell_{1} m_{1}, \ell_{4} m_{4}}\left(C^{-1}\right) \ell_{2} m_{2}, \ell_{5} m_{5}\left(C^{-1}\right)_{\ell_{3} m_{3}, \ell_{6} m_{6}} \\
= & \frac{\alpha}{6} .
\end{aligned}
$$

Comparing with Eq. (A3), (A4), the Monte Carlo expression (Eq. (58)) for $F_{\mathcal{E}}$ follows. 
Finally, from the definition (Eq. (54)), the estimator variance is given by a sum of three terms,

$$
\begin{aligned}
\operatorname{Var}(\mathcal{E}[a])=\frac{1}{F_{\mathcal{E}}}\langle & T\left[C^{-1} a\right] T\left[C^{-1} a\right] \\
& -T\left[C^{-1} a\right]\left(C^{-1} a\right)_{\ell m} \beta_{\ell m} \\
& \left.+\frac{1}{4}\left(C^{-1} a\right)_{\ell_{1} m_{1}} \beta_{\ell_{1} m_{1}}\left(C^{-1} a\right)_{\ell_{2} m_{2}} \beta_{\ell_{2} m_{2}}\right\rangle_{a}
\end{aligned}
$$

which are evaluated as follows:

$$
\begin{aligned}
& \left\langle T\left[C^{-1} a\right] T\left[C^{-1} a\right]\right\rangle_{a} \\
& =\frac{1}{36} B_{\ell_{1} \ell_{2} \ell_{3}} B_{\ell_{4} \ell_{5} \ell_{6}}\left(\begin{array}{ccc}
\ell_{1} & \ell_{2} & \ell_{3} \\
m_{1} & m_{2} & m_{3}
\end{array}\right)\left(\begin{array}{ccc}
\ell_{4} & \ell_{5} & \ell_{6} \\
m_{4} & m_{5} & m_{6}
\end{array}\right) \\
& \times\left\langle\left(C^{-1} a\right)_{\ell_{1} m_{1}}\left(C^{-1} a\right)_{\ell_{2} m_{2}}\left(C^{-1} a\right)_{\ell_{3} m_{3}}\right. \\
& \left.\left(C^{-1} a\right)_{\ell_{4} m_{4}}\left(C^{-1} a\right)_{\ell_{5} m_{5}}\left(C^{-1} a\right)_{\ell_{6} m_{6}}\right\rangle_{a} \\
& =\frac{\alpha}{6}+\frac{1}{4} \beta_{\ell_{1} m_{1}} C_{\ell_{1} m_{1}, \ell_{2} m_{2}}^{-1} \beta_{\ell_{2} m_{2}} \text {. } \\
& \left\langle T\left[C^{-1} a\right]\left(C^{-1} a\right)_{\ell m} \beta_{\ell m}\right\rangle_{a} \\
& =\frac{1}{6} B_{\ell_{1} \ell_{2} \ell_{3}}\left(\begin{array}{ccc}
\ell_{1} & \ell_{2} & \ell_{3} \\
m_{1} & m_{2} & m_{3}
\end{array}\right) \beta_{\ell m} \\
& \times\left\langle\left(C^{-1} a\right)_{\ell_{1} m_{1}}\left(C^{-1} a\right)_{\ell_{2} m_{2}}\left(C^{-1} a\right)_{\ell_{3} m_{3}}\left(C^{-1} a\right)_{\ell m}\right\rangle_{a} \\
& =\frac{1}{2} \beta_{\ell_{1} m_{1}} C_{\ell_{1} m_{1}, \ell_{2} m_{2}}^{-1} \beta_{\ell_{2} m_{2}} \text {. } \\
& \frac{1}{4}\left\langle\left(C^{-1} a\right)_{\ell_{1} m_{1}} \beta_{\ell_{1} m_{1}}\left(C^{-1} a\right)_{\ell_{2} m_{2}} \beta_{\ell_{2} m_{2}}\right\rangle_{a} \\
& =\frac{1}{4} \beta_{\ell_{1} m_{1}} C_{\ell_{1} m_{1}, \ell_{2} m_{2}}^{-1} \beta_{\ell_{2} m_{2}} .
\end{aligned}
$$

Putting Eqs. (A6)-(A9) together, we get

$$
\operatorname{Var}(\mathcal{E})=\frac{\alpha}{6 F_{\mathcal{E}}^{2}}=\frac{1}{F_{\mathcal{E}}}
$$

completing the derivation of Eq. (59).

\section{Appendix B: No $C^{-1}$}

Our Monte Carlo framework for optimal bispectrum estimation (\$VIII) has one experiment-specific requirement: a method for multiplying a CMB map by $C^{-1}$.
In this appendix, we consider the case where this operation is impractical. We will see that it is possible to preserve one feature of the optimal estimator: improving the estimator variance by including the linear term in the bispectrum estimator.

We construct a bispectrum estimator by replacing $C^{-1}$ where it appears in the optimal estimator (Eq. (54) by some filter $F$ which approximates $C^{-1}$ as well as possible:

$$
\mathcal{E}^{\prime}[a]=\frac{1}{F_{\mathcal{E}^{\prime}}}\left(T[F a]-(F a)_{\ell m}\left\langle\nabla_{\ell m} T\left[F a^{\prime}\right]\right\rangle_{a^{\prime}}\right)
$$

where we use primes to distinguish this from the optimal estimator. It can be shown that this choice of linear term minimizes the variance, if the cubic term $T[F a]$ is assumed fixed. (In particular, omitting the linear term from the estimator $\mathcal{E}^{\prime}$ defined above always worsens the variance.) This estimator only depends on the ability to generate simulated signal + noise maps $(F a)$ which are filtered in the same way as the data. Any realistic analysis pipeline can generate such Monte Carlo simulations.

By a calculation parallel to App. A, it can be shown the estimator normalization and variance are given by the following Monte Carlo averages:

$$
\begin{aligned}
F_{\mathcal{E}^{\prime}}= & \frac{1}{3}\left\langle\nabla T[F s] F \nabla T\left[C_{\ell}^{-1} s_{\ell m}\right]\right\rangle_{s} \\
\operatorname{Var}\left(\mathcal{E}^{\prime}\right)= & \frac{1}{3}\left\langle\nabla T[F a] F C F^{T} \nabla T[F a]\right\rangle \\
& \quad-\frac{1}{3}\langle\nabla T[F a]\rangle_{a}\left(F C F^{T}\right)\langle\nabla T[F a]\rangle_{a}
\end{aligned}
$$

Note that the two are not equal, in contrast to the optimal estimator. In the first expression (Eq. ( $\overline{\mathrm{B} 2}$ ) $)$, the Monte Carlo average is taken over signal-only realizations $s$ and the $C_{\ell}^{-1}$ simply refers to division by the signal power spectrum, without reference to the noise model. In the second expression (Eq. (B3)), the average is taken over filtered signal + noise realizations $(F a)$ as usual.

The Monte Carlo prescription given in Eqs. (B2), (B3) does require multiplying CMB maps by $F$ and by $F C F^{T}$. (Note that $F C F^{T}$ is just the covariance matrix of the simulated field $(F a)$.) These operations are easier than multiplying by $C^{-1}$, but in a "pure" Monte Carlo pipeline in which the only possible operation is generating simulations, one can always fall back on direct Monte Carlo evaluations of $\mathcal{E}^{\prime}$ to compute the normalization and variance. (Note that computing the estimator normalization in this way requires non-Gaussian simulations, using the algorithm from $\$[\mathrm{IX}$.) As discussed in \$VIIC, direct Monte Carlo will be slower than a scheme such as Eqs. (B2), (B33).
[1] J. M. Maldacena, JHEP 05, 013 (2003), astro$\mathrm{ph} / 0210603$.

[2] V. Acquaviva, N. Bartolo, S. Matarrese, and A. Riotto, Nucl. Phys. B667, 119 (2003), astro-ph/0209156.
[3] M. Zaldarriaga, Phys. Rev. D69, 043508 (2004), astroph/0306006.

[4] P. Creminelli, JCAP 0310, 003 (2003), astro$\mathrm{ph} / 0306122$. 
[5] N. Arkani-Hamed, P. Creminelli, S. Mukohyama, and M. Zaldarriaga, JCAP 0404, 001 (2004), hepth/0312100.

[6] M. Alishahiha, E. Silverstein, and D. Tong, Phys. Rev. D70, 123505 (2004), hep-th/0404084.

[7] G. I. Rigopoulos, E. P. S. Shellard, and B. W. van Tent, Phys. Rev. D73, 083522 (2006), astro-ph/0506704.

[8] G. Rigopoulos, E. Shellard, and B. van Tent, Phys.Rev. D76, 083512 (2007), astro-ph/0511041.

[9] X. Chen, M.-x. Huang, S. Kachru, and G. Shiu, JCAP 0701, 002 (2007), hep-th/0605045.

[10] X. Chen, R. Easther, and E. A. Lim, JCAP 0706, 023 (2007), astro-ph/0611645.

[11] P. Creminelli, L. Senatore, and M. Zaldarriaga, JCAP 0703, 019 (2007), astro-ph/0606001.

[12] D. Babich, P. Creminelli, and M. Zaldarriaga, JCAP 0408, 009 (2004), astro-ph/0405356.

[13] P. Creminelli and M. Zaldarriaga, JCAP 0410, 006 (2004), astro-ph/0407059.

[14] D. N. Spergel and D. M. Goldberg, Phys. Rev. D59, 103001 (1999), astro-ph/9811252.

[15] D. M. Goldberg and D. N. Spergel, Phys. Rev. D59, 103002 (1999), astro-ph/9811251.

[16] L. Verde and D. N. Spergel, Phys. Rev. D65, 043007 (2002), astro-ph/0108179.

[17] A. R. Cooray and W. Hu, Astrophys. J. 534, 533 (2000), astro-ph/9910397.

[18] E. Komatsu, D. N. Spergel, and B. D. Wandelt, Astrophys. J. 634, 14 (2005), astro-ph/0305189.

[19] P. Creminelli, A. Nicolis, L. Senatore, M. Tegmark, and M. Zaldarriaga, JCAP 0605, 004 (2006), astroph/0509029.

[20] J. R. Bond, A. H. Jaffe, and L. Knox, Phys. Rev. D57, 2117 (1998), astro-ph/9708203.

[21] B. D. Wandelt and F. K. Hansen, Phys. Rev. D67, 023001 (2003), astro-ph/0106515.

[22] S. P. Oh, D. N. Spergel, and G. Hinshaw, Astrophys. J. 510, 551 (1999), astro-ph/9805339.

[23] B. D. Wandelt, E. Hivon, and K. M. Gorski, Phys. Rev. D64, 083003 (2001), astro-ph/0008111.

[24] I. Szapudi, S. Prunet, D. Pogosyan, A. S. Szalay, and J. R. Bond, Astrophys.J.Lett. 548, 115 (2001), astro$\mathrm{ph} / 0010256$.

[25] E. Hivon, K. Gorski, C. Netterfield, B. Crill, S. Prunet, et al., Astrophys.J. 567, 2 (2002), * Brief entry *, astro$\mathrm{ph} / 0105302$.

[26] G. Efstathiou, Mon. Not. Roy. Astron. Soc. 349, 603 (2004), astro-ph/0307515.

[27] B. D. Wandelt, D. L. Larson, and A. Lakshminarayanan, Phys. Rev. D70, 083511 (2004), astro-ph/0310080.

[28] J. Jewell, S. Levin, and C. H. Anderson, Astrophys. J. 609, 1 (2004), astro-ph/0209560.

[29] E. Komatsu et al., Astrophys. J. Suppl. 148, 119 (2003), astro-ph/0302223.

[30] M. Liguori, S. Matarrese, and L. Moscardini, Astrophys. J. 597, 57 (2003), astro-ph/0306248.
[31] C. R. Contaldi and J. Magueijo, Phys. Rev. D63, 103512 (2001), astro-ph/0101512.

[32] G. Rocha, M. P. Hobson, S. Smith, P. Ferreira, and A. Challinor, Mon. Not. Roy. Astron. Soc. 357, 1 (2005), astro-ph/0406136.

[33] D. Babich and M. Zaldarriaga, Phys. Rev. D70, 083005 (2004), astro-ph/0408455.

[34] E. Komatsu and D. N. Spergel, Phys. Rev. D63, 063002 (2001), astro-ph/0005036.

[35] E. Komatsu (2002), astro-ph/0206039.

[36] W. Hu, Phys. Rev. D62, 043007 (2000), astro$\mathrm{ph} / 0001303$.

[37] U. Seljak and M. Zaldarriaga, Phys. Rev. D60, 043504 (1999), astro-ph/9811123.

[38] W. Hu, Astrophys. J. 529, 12 (2000), astro-ph/9907103.

[39] L. Toffolatti et al., Mon. Not. Roy. Astron. Soc. 297, 117 (1998), astro-ph/9711085.

[40] L.-M. Wang and M. Kamionkowski, Phys. Rev. D61, 063504 (2000), astro-ph/9907431.

[41] M. Liguori, F. K. Hansen, E. Komatsu, S. Matarrese, and A. Riotto, Phys. Rev. D73, 043505 (2006), astroph/0509098.

[42] W. H. Press, B. P. Flannery, S. A. Teukolsky, and W. T. Vetterling, Numerical Recipes: The Art of Scientific Computing (Cambridge University Press, Cambridge (UK) and New York, 1992), 2nd ed., ISBN 0-52143064-X.

[43] A. Albrecht et al. (2006), astro-ph/0609591.

[44] P. Creminelli, L. Senatore, M. Zaldarriaga, and M. Tegmark, JCAP 0703, 005 (2007), astro-ph/0610600.

[45] J. Borrill (1998), astro-ph/9803114.

[46] D. Babich, Phys. Rev. D72, 043003 (2005), astro$\mathrm{ph} / 0503375$.

[47] D. Spergel et al. (WMAP Collaboration), Astrophys.J.Suppl. 170, 377 (2007), astro-ph/0603449.

[48] A. Doroshkevich, P. Naselsky, O. V. Verkhodanov, D. Novikov, V. Turchaninov, et al., Int. J. Mod. Phys. D. 14, 275 (2005), astro-ph/0305537.

[49] D. Hanson, K. M. Smith, A. Challinor, and M. Liguori, Phys. Rev. D80, 083004 (2009), 0905.4732.

[50] P. Creminelli and M. Zaldarriaga, Phys. Rev. D70, 083532 (2004), astro-ph/0405428.

[51] This form of the bispectrum is problematic as it does not satisfy a simple consistency condition resulting from causality requirements [13, 50]. The bispectrum does not vanish when one takes the limit of one mode being very large compared to the horizon. We will ignore this problem here as we are just taking it as an illustrative example that has been recently analyzed in detain in the literature. We will later find that this shape is very correlated with a linear combination of the local and equilateral shapes. The correlation with the local shape is probably unphysical, originating from the fact that this shape does not satisfy the appropriate consistency relation. 